\title{
IDENTIFICATION OF INFORMATION REQUIREMENTS FOR IMPLEMENTING BUILDING INFORMATION MODELING BASED ON MODEL USES
}

\author{
SUBMITTED: May 2020 \\ REVISED: November 2020 \\ PUBLISHED: December 2020 \\ EDITOR: Žiga Turk \\ DOI: $10.36680 /$ j.itcon.2020.032
}

Raif Alshorafa, Ph.D. Candidate,

Department of Civil Engineering, Istanbul Technical University, Istanbul, Turkey, ralshorafa@itu.edu.tr

\author{
Esin Ergen, Professor, \\ Department of Civil Engineering, Istanbul Technical University, Istanbul, Turkey, \\ esin.ergen@itu.edu.tr
}

\begin{abstract}
SUMMARY: This study aimed to provide guidance in adopting BIM, specifically in determining the level of development of the components in a model. The main objective was to identify the information items to be included in a model based on the required BIM uses. Semi-structured face-to-face interviews were conducted with nine professionals to identify the information items that are required for the selected BIM uses. The professionals filled out a form by selecting the required information items from a list of component properties for specific BIM uses. The responses were analyzed to determine the most frequently selected information items for each BIM use. The results were validated via literal replication and external validation. The main properties that were most frequently selected were determined for two types of building components, duct and pipe, and limited to five BIM uses. The study revealed that the most frequently selected properties and related BIM modeling effort varied based on the BIM use/s required in different project types. The list of identified information items can be used by the practitioners when defining the properties required to prepare a BIM model for the selected BIM uses. The practitioners can utilize the findings of this study to increase their efficiency in adopting BIM and decrease the time and effort loss and cost overruns while adopting BIM. Although the previous studies emphasized that determination of the right level of development from the very beginning of the construction process is critical, guidance on which information items are required are lacking in the literature. This study provided guidance on how the required properties of elements that are going to be included in a BIM model changes based on BIM uses. Future studies can investigate the information needs required for other types of components and for other BIM uses.
\end{abstract}

KEYWORDS: Building Information Modeling, BIM uses, Level of Development, LOD.

REFERENCE: Raif Alshorafa, Esin Ergen (2020). Identification of information requirements for implementing Building Information Modeling based on model uses. Journal of Information Technology in Construction (ITcon), Vol. 25, pg. 561-574, DOI: 10.36680/j.itcon.2020.032

COPYRIGHT: (C) 2020 The author(s). This is an open access article distributed under the terms of the Creative Commons Attribution 4.0 International (https://creativecommons.org/licenses/by/4.0/), which permits unrestricted use, distribution, and reproduction in any medium, provided the original work is properly cited. 


\section{INTRODUCTION}

Building Information Modeling (BIM) involves creating and maintaining an integral digital representation of building information through the project life cycle in the form of a data repository, including geometric and nongeometric data (Gu \& London, 2010). Previous research shows that the adoption rate of BIM is slow in the AEC industry (Liu et al., 2017 and Herr \& Fischer, 2019), and the degree of integration of BIM with standards varies extensively (Mzyece et al., 2019). One of the reasons is related to the intense and experience-oriented planning and preparation that is required before implementing BIM (Eadie et al., 2013; Liu et al., 2017 and Pezeshki \& Ivari, 2018). Since construction projects are unique, transferring knowledge from one project to another one is challenging (Olawumi \& Chan, 2019). Moreover, the accumulated knowledge in BIM adoption, which is a relatively emerging concept, is not well documented and the informal BIM implementation processes do not support knowledge capture (Jung \& Joo, 2011; Wang \& Leite, 2015 and Wang \& Meng, 2018). Consequently, a well-established systematic BIM-based workflow is crucial, and more guidance is required for BIM implementation (Gu \& London, 2010; Leite et al., 2011 \& Tan et al., 2019).

An important component of BIM implementation is the definition of Level of Development (LOD) of the model. LOD describes the information items (i.e., geometric and non-geometric) to be included in a BIM model (Ahmad Latiffi et al., 2015). Based on the defined LOD, the project components can be represented in a BIM model from the lowest level of approximation to the highest level of representation (Leite et al., 2011).

LOD was first defined by The American Institute of Architects (AIA) in 2008 (AIA, 2008) and updated in 2013 (AIA, 2013). BIMForum later extended the AIA's LOD definitions by including examples to illustrate the interpretations of LOD definitions (BIMForum, 2019). Finally, ISO recently published two BIM standards related to managing information over the whole life cycle of a built asset (ISO, 2018). In ISO 19650-1, Level of Information Need (LOIN) was defined as "a framework which defines the extent and granularity of information". It was indicated that "LOIN of each information deliverable should be determined according to its purpose" and a variety of metrics can be used to determine LOIN (e.g., LOD) (ISO, 2018).

Defining the right LOD from the very beginning of the construction process is critical because of its vast influence on the progression of BIM implementation (Leite et al., 2011). Missing information results in rework required for updating the model (Alshorafa \& Ergen, 2019), whereas the provision of too much information is considered waste (ISO, 2018). Despite this known importance, reaching an agreement about LOD/LOIN before implementation is not a standard process in practice (Biljecki et al., 2016; Charef et al., 2018 \& Tan et al., 2019). The parties usually define the LOD in the contract and they are expected to elaborate the information need in detail in the BIM Execution Plan (BEP), which describes the vision of implementation details to be followed by the team throughout the project stages (CIC, 2011). However, a previous study conducted by the authors showed that although the LOD was contractually defined in the four investigated cases, the parties had difficult times in reaching a level of agreement about the details of information needs and most of them did not include detailed information in BEP. Thus, additional needs and requirements by the client/owner were being defined through the project (Alshorafa \& Ergen, 2019).

Previous studies showed that a consensus even on the definition of LOD was lacking due to vague terms used in the LOD definitions (Van Berlo et al., 2014 \& Biljecki et al., 2016). Defining the required information items is especially crucial for non-geometric information since the users need to select the information items from a long list of items based on the determined BIM uses. Since the priority is given to defining the geometric information in a model in practice, ISO standard also pointed out that the granularity of non-geometric information is as significant as that of geometric information (ISO, 2018). The previous studies and the ISO standard emphasized that LOD should be defined based on the needs of the project by the project parties (Leite et al., 2011; Van Berlo et al., 2014, ISO, 2018; Hong et al., 2019 \& BIMForum, 2019); however, there is no guidance to define which project needs require what information items to be incorporated in the model.

This study aims to identify the information items to be included in a model based on the required BIM uses in order to provide guidance in adopting BIM. A qualitative study was performed to conduct face-to-face interviews and to collect expert opinions via a data collection form. Nine practitioners who have experience in large-scale projects participated in the study. The emphasis was on large-scale projects because of the complexity of information exchange among numerous disciplines and parties involved in the process. The authors specifically focused on the information items required in the construction phase for two types of components, pipes and ducts, 
since the information items related to mechanical components are more detailed and these components are commonly used in most project types. It is envisioned that the identified information items and the related BIM uses will provide guidance to the practitioners to define LOD of components in a project before implementation and minimize the current limitations of BIM adoption (Alshorafa \& Ergen, 2019).

\section{LITERATURE REVIEW}

BIM concepts and technologies are being discussed and implemented for the last two decades (Lu et al., 2017), it is still considered as an emerging approach (Lu et al., 2017 and Ghannadpour et al., 2019). BIM is being adopted not only in building construction but also in infrastructure and industrial projects. Although initial BIM adoption was observed in the building construction, the infrastructure industry/sector is rapidly catching up with the building sector in BIM adoption due to observed benefits in the building construction. Moreover, BIM adoption rate among contractors in infrastructure projects follows building projects consistently across all regions. BIM adoption in infrastructure projects was identified as a globally growing area according to The Smart Market Report (2014), and ranks second after industrial/manufacturing non-building project types adopting BIM (McGraw-Hill Construction, 2014). The infrastructure projects are usually large-scale and have MEP intensive components; thus, the benefits gained from the utilization of BIM are significant. These projects also have many similarities with the building projects including numerous common components and similar approaches that are used in BIM implementation. Ibrahim et al. (2013) highlighted the similarity in using collaboration technologies in major building and infrastructure projects. In specific situations, these technologies are grouped and applied in similar ways for both building and infrastructure projects (Ibrahim et al., 2013). Shou et al., (2015) stated that the BIM application in infrastructure projects was dependent on accumulated experience in building projects (Shou et al., 2015). Chong et al., (2016) stated that both project types, i.e. buildings and infrastructure, had almost the same BIM uses, associated technologies, key persons' roles, and responsibilities in their comparative study.

The adoption rate of BIM is evaluated as slow in the AEC industry due to multiple reasons (Liu et al., 2017 and Herr \& Fischer, 2019). For example, the need for intense training before BIM adoption (Arayici et al., 2011 and Eadie et al., 2013), high dependency on experience for implementation (Liu et al., 2017; Olawumi \& Chan, 2017; Alreshidi et al., 2017 and Pezeshki \& Ivori, 2018) and the requirement for continuous and efficient collaboration environment (Singh et al., 2011; Eadie et al., 2013; Liu et al., 2017; Alreshidi et al., 2017; Lai \& Deng, 2018 \& Alreshidi et al., 2018).

Previous studies investigated BIM from various perspectives, such as BIM maturity (Succar, 2009; Dakhil et al., 2019 and Abualdenien \& Borrmann, 2019), BIM capacities or uses (Ghannadpour et al., 2019), BIM policies (Kassem \& Succar, 2017), decision making and governance (Singh et al., 2011; Nawari, 2012; Alreshidi et al., 2017; Alreshidi et al., 2018; Olawumi \& Chan, 2019 and Abualdenien \& Borrmann, 2019), and collaboration, interoperability, and integrity (Vanlande et al., 2008; Singh et al., 2011; Eadie et al., 2013; Liu et al., 2017; Lai \& Deng, 2018 and Alreshidi et al., 2018). Yet, only a few studies focused on the Level of Development (LOD), although it is a crucial part of BIM adoption process as the early definition of the correct LOD will influence the progression of BIM implementation process in the following stages of the project (Leite et al., 2011). LOD was first defined in 2008 by the AIA, and the definition was later updated as: "The LOD describes the minimum dimensional, spatial, quantitative, qualitative, and other data included in a Model Element to support the Authorized Uses associated with such LOD” (AIA, 2013).

Five LODs were defined by the AIA in 2008, and these basic definitions were elaborated by The BIMForum Working Group to interpret the original definitions. BIMForum also highlighted this fact emphasizing that a single LOD cannot be defined for an entire model, stating that: "There is no such thing as a (LOD \#\#\# model)." (BIMForum, 2019). The BIMForum LOD Specification provided examples to describe geometric and nongeometric information for major building elements at each LOD. A geometric representation of specific model elements and attribute lists at each LOD was presented. Although this specification was more detailed, it still lacks guidance as to which specific information items should be included in the model based on needs/BIM uses since only mandatory and optional information items were provided. ISO 19650-1, introduced a new term called Level of Information Need (LOIN) to define the extent and granularity of information (ISO, 2018). It was indicated that LOIN can still be defined based on a LOD standard or other modeling guidelines (e.g., BIMForum LOD Specification) (ISO, 2018 \& EFCA, 2019). 
Similar to BIMForum LOD Specification, the Construction Operations Building Information Exchange (COBie) standard supplies the list of information items (i.e. non-geometric information) that should be collected during design and construction phases to be delivered to the employer or operator for use in the facility management phase (East, 2007 and BSI, 2013). However, it is developed for facility management and the user needs to select the required information items based on specific requirements. Other guidelines also include recommendations on the LOD definition process; yet, they are usually generic (Eastman et al., 2008; CIC, 2011; Department of VA, 2010 and CA Singapore, 2013).

The review of current LOD and LOIN definitions shows that there is no guidance to determine what information should be included in a BIM model for different BIM uses and the parties involved in the project should define it collaboratively. The available standards require highly accumulated experience and understanding of the entire BIM uses implemented through the life cycle of the project. The BIM implementation remains a manual process (entered by user or modeler) (Abualdenien et al., 2019). The researchers emphasized that automating the use of BIM related standards under a user-friendly graphical interface could improve governance, facilitate team collaboration, and enhance decision-making during construction projects (Nawari, 2012; Alreshidi et al., 2017 and Yalcinkaya \& Singh, 2019). However, the information items to be included in the model should still be identified based on BIM uses to provide guidance in using those tools.

\section{METHODOLOGY}

A qualitative study with a triangulation approach was adopted in this study. The triangulation approach was used to observe a researched issue from at least two different perspectives (Flick et al., 2004). Semi-structured face-toface in-depth interviews were conducted with nine participants for data collection. Interviews include open-ended questions and include rich discussions; therefore, the data collected in the interviews enable in-depth analysis of the problem at hand (Johnson, 1997; Kothari, 2004 \& Boddy, 2016). To capture varying opinions of different groups of practitioners working in different project types, three groups of respondents having three persons each participated in the study, and their responses were triangulated in two stages. In the first stage, the responses of three respondents in each group were triangulated to determine the group's response. In the second stage, the responses of the three groups were triangulated to determine the overall response of the respondents.

The study focused on the information needs in the construction phase. To determine which BIM uses, building components and properties should be focused on in the scope of this study, initial meetings and brainstorming sessions were performed with three experts (i.e., two BIM consultants and one BIM manager). The experts were chosen based on the criteria of having at least ten years of experience with BIM implementation in a variety of project types including both residential/commercial and transportation/infrastructure projects. Afterward, semistructured face-to-face interviews were conducted with nine professionals and a data collection form was employed to collect data from the respondents. The responses were triangulated in two stages to identify the most frequently selected information requirements for the selected building components and BIM uses. The results were validated via literal replication and external validation.

Before the initial interviews, the following BIM guides, standards and codes of practice were investigated to select the major BIM uses to be utilized in this study: Level of Development Specification Guide, BIM Handbook, The Singapore BIM Guide, The Veterans Affairs BIM Guide, BIM Project Execution Planning Guide, and Document E202-2008 Building Information Modeling Protocol Exhibit (Eastman et al., 2008; AIA, 2008; Department of VA, 2010; CIC, 2011; BCA Singapore, 2013 and BIMForum, 2019). A list of 22 BIM uses was selected from those resources and presented to the experts. Among them, five BIM uses were selected by the experts as the most common BIM uses that should be included in the study. In the second step, the model components to be focused on in this study were identified. The experts selected duct and pipe components because information items related to mechanical components are more detailed, and these mechanical components are the most commonly used components among various project types. Finally, to determine all the properties that are related to these components, three lists that describe the building components and their related properties were investigated: 1) The US national BIM standard lists (3M matrix) (NBIMS, 2015) 2) The Australian NATSPEC BIM Object Element Matrix (NATSPEC, 2011), and 3) The BIMForum 2017 lists of components (BIMForum, 2017). The lists included in these standards were reviewed and compared to find if the properties of components that were provided in the standards could be used in the data collection tool. These standards include a wide range of information that was required for different construction components in different stages. The National BIM Standard provided a 
Minimum Modeling Matrix that categorizes the building components and no detailed properties were given for the elements in this list (NBIMS, 2015). The NATSPEC lists contained 15 information categories (e.g., physical properties, manufacturer, specifications, facility management) that include properties for 27 building components. The list of properties are extensive, (i.e., about 280 properties for Mechanical Equipment at LOD500) (NATSPEC, 2011). In the BIMForum Lists, both global attributes and item-specific attributes were provided for 39 building components, with their description and their corresponding LOD. However, these attributes are not inclusive. For example, the "Air Distribution category" contained information about the duct component, which was almost entirely considered in the Revit properties; however, the properties related to installation, scheduling, or facility management (which were included in Revit) were not listed (BIMForum, 2017).

The investigation of existing standards showed that they are either extensive or not inclusive. In addition, the targeted respondents were not familiar with these standard lists, and it would be challenging for them to understand a long list of items before filling the data collection tool. Therefore, since Revit was a commonly used BIM authoring tool in the AEC sector (McGraw-Hill Construction, 2014) and the respondents were more familiar with them, the experts recommended that the properties in Autodesk Revit ${ }^{\circledR} 2017$ to be used for the selected MEP components.

Once the BIM uses, the components to be investigated, and their properties were selected, the respondents' opinions were collected on the information items/properties that were required for each BIM use and component. The theoretical purposive sampling approach was adopted in selecting the respondents. The respondents were chosen based on particular qualifications that enable detailed exploration and understanding of the central themes in the study and generalization of the findings (Ritchie et al., 2013 \& Yin, 2011). The main criterium that was used in selecting the participants was to have previous BIM experience in two different types of projects, which are housing/commercial buildings or transportation/infrastructure projects. As indicated in the literature review section, these two types of projects have many similarities including numerous common components and similar approaches that are used in BIM implementation. By focusing on these two types of projects, the goal was to identify the similarities and differences in them. Using this criterium will support the assumption of the generality of the study in different project types. Table (1) shows the criteria of the participants in the study.

Table (1): characteristics of the sample of respondents.

\begin{tabular}{|l|l|l|l|l|}
\hline Group & Respondent & Position & Firm Type & Current Project \\
\hline \multirow{3}{*}{ Group 1 } & 1 & BIM manager & Contractor & Railway Project \\
\cline { 2 - 3 } & 2 & Senior MEP specialist & & \\
\cline { 2 - 3 } & 3 & BIM Modeler & & \\
\hline \multirow{3}{*}{ Group 2 } & 4 & BIM coordinator & Design Firm & Metro Line \\
\cline { 2 - 3 } & 5 & BIM Engineer & Contractor & Airport \\
\cline { 2 - 3 } & 6 & BIM Coordinator & Contractor & \\
\cline { 2 - 3 } & 7 & BIM consultant & BIM Consulting & Residential \& commercial composite \\
\cline { 2 - 3 } & 8 & BIM consultant & & \\
\hline
\end{tabular}

Semi-structured face-to-face interviews were conducted with the experts and they were asked to fill a form (Tables $3 \& 4)$ to indicate which properties of the selected components are needed for performing each BIM use. Each interview lasted for about two hours each to clarify the aim of the study and how to fill the data collection form adequately. In some cases, one of the authors assisted respondents to fill out the data collection form by providing discussions and explanations. The need for 58 and 56 properties of two building components (i.e., duct and pipe) were determined by the respondents for five BIM uses (i.e. each of the properties was evaluated five times by each respondent). When analyzing the answers, if there were any missing or contradicting data, the respondents were contacted again for clarification. Therefore, each interview includes extensive discussions, which lead to the findings of the study.

The results were triangulated based on the frequency of selection to identify the most frequently selected information items for each BIM use. To validate the results of the study, literal replication and external validation were performed. Literal replication is replicating the results of a study with another study conducted with a similar group of participants (Yin, 2011). External validation is to find out if the results of a specific test can be sufficiently replicated in similar situations so that the findings can be generalized to other similar situations (Yin, 2011). To perform literal replication and external validation, the respondents were divided into three groups having three 
participants each with varying levels of experience. The first two groups consisted of practitioners that have previous experience in transportation/infrastructure projects, and the literal replication method was used to determine the similarity between the results of these two groups of respondents. The third group contained practitioners with previous experience in a different area, which is housing/commercial building projects. The results of the first and second groups were compared with this third group to test external validation of the responses between groups working on different types of projects (Yin, 2011).

\section{ANALYSIS AND FINDINGS:}

The data was collected via a data collection form that was created for two components (i.e., duct and pipe). Each table had 1) five selected BIM uses and 2) nine categories of properties for duct and eight categories of properties for pipe components. Each respondent was asked to fill out these two tables to select the properties that should be included in the model for each BIM use.

The selected BIM uses were clash detection (Clsh), 4D scheduling (4D), quantity take-off (QTO), shop drawing $(\mathrm{ShD})$, and facility management (FM). 56 properties were included for both duct and pipe under eight categories and one additional category having two properties (i.e. lining properties) was defined for the duct component (Tables $3 \& 4$ ). The Additional Information category was added by the authors based on the experts' recommendations and initial discussions. These categories of properties are described in Table 2.

Table (2): Categories of properties.

\begin{tabular}{|c|l|l|l|}
\hline No. & Main groups of properties & \multicolumn{1}{|c|}{ Description } & \multicolumn{1}{|c|}{ Examples of properties } \\
\hline 1 & Constraints. & Indicates location and justification properties. & Reference level \& slope. \\
\hline 2 & Mechanical information. & Describes mechanical properties and variables. & Elevation and loss coefficient. \\
\hline 3 & $\begin{array}{l}\text { Mechanical-flow } \\
\text { information. }\end{array}$ & Consists of variables related to the mechanical properties. & Velocity and friction. \\
\hline 4 & Dimensions. & Describes basic dimensional information. & Size and diameter. \\
\hline 5 & Identity information. & Indicates mainly contextual information related to the component. & Model, manufacturer, images. \\
\hline 6 & Insulation. & Describes the insulation. & Type, thickness and size. \\
\hline 7 & Accessories. & Indicates information related to accessories and fixtures & Place holders and fittings. \\
\hline 8 & Additional information. & $\begin{array}{l}\text { Added by the authors based on the initial brainstorming sessions } \\
\text { with practitioners. }\end{array}$ & $\begin{array}{l}\text { WBS code, BOQ code, installation date, commission date, } \\
\text { and warranty period. }\end{array}$ \\
\hline 9 & Lining (for Duct only). & Describing properties related to the type and size of ducts only. & Free size and lining thickness and type. \\
\hline
\end{tabular}

The collected data was analyzed to determine the frequency of selected information items by the respondents, which were divided into three groups based on their background, namely G1, G2 (i.e. transportation/infrastructure), and G3 (residential \& commercial composite) (Table 1). Table 3 presents the most frequently selected information items that are determined based on their frequency for five BIM uses for ducts and Table 4 presents the most selected information items for pipes.

Table (3): Most frequently selected properties for ducts.

\begin{tabular}{|c|c|c|c|c|c|c|c|c|c|c|c|c|c|c|c|c|c|c|c|c|c|}
\hline \multicolumn{2}{|c|}{ Properties } & \multicolumn{10}{|c|}{$\begin{array}{l}\text { Mutual 2-3 answers to be considered by } \\
\qquad \mathrm{G} 1+\mathrm{G} 2\end{array}$} & \multicolumn{5}{|c|}{$\begin{array}{c}\text { Mutual 2-3 answers to be } \\
\text { considered by } \\
\text { G3 }\end{array}$} & \multicolumn{5}{|c|}{$\begin{array}{c}\text { Required information } \\
\text { items } \\
(\mathrm{G} 1+\mathrm{G} 2)+\mathrm{G} 3\end{array}$} \\
\hline \# & Duct Item & \multicolumn{2}{|c|}{ Clsh } & \multicolumn{2}{|c|}{$4 \mathrm{D}$} & \multicolumn{2}{|c|}{ QTO } & \multicolumn{2}{|c|}{ ShD } & \multicolumn{2}{|c|}{ FM } & \multicolumn{2}{|c|}{ Clsh $4 \mathrm{D}$} & QTO & \multicolumn{2}{|c|}{\begin{tabular}{|l|l|} 
ShD & FM \\
\end{tabular}} & \multirow{3}{*}{\multicolumn{5}{|c|}{ 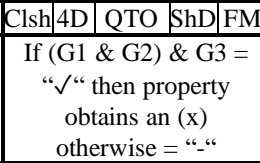 }} \\
\hline & Duct Type (Rect. - Circ. - Oval - Flex) & G1 & $\mathrm{G} 2$ & G1 & $\mathrm{G} 2$ & G1 & $\mathrm{G} 2$ & G1 & G2 & G1 & $\mathrm{G} 2$ & \multirow{2}{*}{\multicolumn{5}{|c|}{$\begin{aligned} 2 / 3 & =3 / 3=“ \sqrt{ }= \\
0 / 3 & =1 / 3=“-“\end{aligned}$}} & & & & & \\
\hline 1 & Constraints & & $\begin{array}{l}(2 / 3 \\
20 / 3)\end{array}$ & $\& 2 / 3)$ & $=(2 / 3$ & $3 / 3$ & )$=(3$ & $3 \&$ & (3) $=$ & & & & & & & & & & & & \\
\hline 1.1 . & Horizontal Justification & $\checkmark$ & $\checkmark$ & $\checkmark$ & $\checkmark$ & $\checkmark$ & $\checkmark$ & $\checkmark$ & $\checkmark$ & $\checkmark$ & $\checkmark$ & $\checkmark$ & $\checkmark$ & $\checkmark$ & $\checkmark$ & $\checkmark$ & $\mathrm{x}$ & $\mathrm{x}$ & $\mathrm{x}$ & $\mathrm{x}$ & $\mathrm{x}$ \\
\hline 1.2 . & Vertical Justification & $\checkmark$ & $\checkmark$ & $\checkmark$ & $\checkmark$ & $\checkmark$ & $\checkmark$ & $\checkmark$ & $\checkmark$ & $\checkmark$ & $\checkmark$ & $\checkmark$ & $\checkmark$ & $\checkmark$ & $\checkmark$ & $\checkmark$ & $\mathrm{x}$ & $\mathrm{x}$ & $\mathrm{x}$ & $\mathrm{x}$ & $\mathrm{x}$ \\
\hline 1.3 . & Reference level & $\checkmark$ & $\checkmark$ & $\checkmark$ & $\checkmark$ & $\checkmark$ & $\checkmark$ & $\checkmark$ & $\checkmark$ & $\checkmark$ & $\checkmark$ & $\checkmark$ & $\checkmark$ & $\checkmark$ & $\checkmark$ & $\checkmark$ & $\mathrm{x}$ & $\mathrm{x}$ & $\mathrm{x}$ & $\mathrm{x}$ & $\mathrm{x}$ \\
\hline 1.4 . & Offset within floor & $\checkmark$ & $\checkmark$ & $\checkmark$ & $\checkmark$ & $\checkmark$ & $\checkmark$ & $\checkmark$ & $\checkmark$ & $\checkmark$ & $\checkmark$ & $\checkmark$ & $\checkmark$ & $\checkmark$ & $\checkmark$ & $\checkmark$ & $\mathrm{x}$ & $\mathrm{x}$ & $\mathrm{x}$ & $\mathrm{x}$ & $\mathrm{x}$ \\
\hline 1.5 . & Start Offset & $\checkmark$ & $\checkmark$ & $\checkmark$ & $\checkmark$ & $\checkmark$ & $\checkmark$ & $\checkmark$ & $\checkmark$ & $\checkmark$ & $\checkmark$ & $\checkmark$ & $\checkmark$ & $\checkmark$ & $\checkmark$ & $\checkmark$ & $\mathrm{x}$ & $\mathrm{x}$ & $\mathrm{x}$ & $\mathrm{x}$ & $\mathrm{x}$ \\
\hline 1.6. & End Offset & $\checkmark$ & $\checkmark$ & $\checkmark$ & $\checkmark$ & $\checkmark$ & $\checkmark$ & $\checkmark$ & $\checkmark$ & $\checkmark$ & $\checkmark$ & $\checkmark$ & $\checkmark$ & $\checkmark$ & $\checkmark$ & $\checkmark$ & $\mathrm{x}$ & $\mathrm{x}$ & $\mathrm{x}$ & $\mathrm{x}$ & $\mathrm{x}$ \\
\hline 1.7 . & Slope & $\checkmark$ & $\checkmark$ & $\sqrt{ }$ & $\checkmark$ & $\checkmark$ & $\checkmark$ & $\checkmark$ & $\checkmark$ & $\checkmark$ & $\checkmark$ & $\checkmark$ & $\checkmark$ & $\sqrt{ }$ & $\checkmark$ & $\checkmark$ & $\mathrm{x}$ & $\mathrm{x}$ & $\mathrm{x}$ & $\mathrm{x}$ & $\mathrm{x}$ \\
\hline 2 & Mechanical & & & & & & & & & & & & & & & & & & & & \\
\hline 2.1. & System Classification & $\checkmark$ & $\checkmark$ & $\checkmark$ & $\checkmark$ & $\checkmark$ & $\checkmark$ & $\checkmark$ & $\checkmark$ & $\checkmark$ & $\checkmark$ & $\checkmark$ & $\checkmark$ & $\checkmark$ & $\checkmark$ & $\checkmark$ & $\mathrm{x}$ & $\mathrm{x}$ & $\mathrm{x}$ & $\mathrm{x}$ & $\mathrm{x}$ \\
\hline 2.2 . & System Type & $\checkmark$ & $\checkmark$ & $\checkmark$ & $\checkmark$ & $\checkmark$ & $\checkmark$ & $\checkmark$ & $\checkmark$ & $\checkmark$ & $\checkmark$ & $\checkmark$ & $\checkmark$ & $\checkmark$ & $\checkmark$ & $\checkmark$ & $\mathrm{x}$ & $\mathrm{x}$ & $\mathrm{x}$ & $\mathrm{x}$ & $\mathrm{x}$ \\
\hline 2.3 . & System Abbreviation & $\checkmark$ & $\checkmark$ & $\checkmark$ & $\checkmark$ & $\checkmark$ & $\checkmark$ & $\checkmark$ & $\checkmark$ & $\checkmark$ & $\checkmark$ & $\checkmark$ & $\checkmark$ & $\checkmark$ & $\checkmark$ & $\checkmark$ & $\mathrm{x}$ & $\mathrm{x}$ & $\mathrm{x}$ & $\mathrm{x}$ & $\mathrm{x}$ \\
\hline 2.4 . & Bottom Elevation & $\checkmark$ & $\checkmark$ & $\checkmark$ & $\checkmark$ & $\checkmark$ & $\checkmark$ & $\checkmark$ & $\checkmark$ & $\checkmark$ & $\checkmark$ & $\checkmark$ & $\checkmark$ & $\checkmark$ & $\checkmark$ & $\checkmark$ & $\mathrm{x}$ & $\mathrm{x}$ & $\mathrm{x}$ & $\mathrm{x}$ & $\mathrm{x}$ \\
\hline
\end{tabular}




\begin{tabular}{|c|c|c|c|c|c|c|c|c|c|c|c|c|c|c|c|c|c|c|c|c|c|}
\hline \multirow{3}{*}{\multicolumn{2}{|c|}{\begin{tabular}{|c|c|}
\multicolumn{2}{|c}{ Properties } \\
$\#$ & \multicolumn{1}{|c}{ Duct Item } \\
2.5. & Top Elevation
\end{tabular}}} & \multicolumn{10}{|c|}{$\begin{array}{l}\text { Mutual 2-3 answers to be considered by } \\
\text { G1+G2 }\end{array}$} & \multicolumn{5}{|c|}{$\begin{array}{c}\text { Mutual } 2-3 \text { answers to be } \\
\text { considered by } \\
\text { G3 }\end{array}$} & \multicolumn{5}{|c|}{$\begin{array}{c}\text { Required information } \\
\text { items } \\
(\mathrm{G} 1+\mathrm{G} 2)+\mathrm{G} 3\end{array}$} \\
\hline & & \multicolumn{2}{|c|}{ Clsh } & \multicolumn{2}{|c|}{$4 \mathrm{D}$} & \multicolumn{2}{|c|}{ QTO } & \multicolumn{2}{|c|}{$\mathrm{ShD}$} & \multicolumn{2}{|c|}{ FM } & \multicolumn{5}{|c|}{\begin{tabular}{|l|l|l|l|l|} 
Clsh & 4D & QTO & ShD & FM \\
\end{tabular}} & & $4 \mathrm{D}$ & QTO & & FM \\
\hline & & $\checkmark$ & $\checkmark$ & $\checkmark$ & $\checkmark$ & $\checkmark$ & $\checkmark$ & $\checkmark$ & $\checkmark$ & $\checkmark$ & $\checkmark$ & $\mid \sqrt{ }$ & $\checkmark$ & $\checkmark$ & $\checkmark$ & $\checkmark$ & $\mathrm{x}$ & $\mathrm{x}$ & $\mathrm{x}$ & $\mathrm{x}$ & $\mathrm{x}$ \\
\hline 2.6. & Equivalent Diameter & $\checkmark$ & $\checkmark$ & $\checkmark$ & $\checkmark$ & $\checkmark$ & $\checkmark$ & $\checkmark$ & $\checkmark$ & $\checkmark$ & $\checkmark$ & $\checkmark$ & $\checkmark$ & $\checkmark$ & $\checkmark$ & $\checkmark$ & $\mathrm{x}$ & $\mathrm{x}$ & $\mathrm{x}$ & $\mathrm{x}$ & $\mathrm{x}$ \\
\hline 2.7. & Loss Coefficient & - & - & - & - & - & - & - & - & - & $\checkmark$ & - & - & - & - & - & - & - & - & - & - \\
\hline 2.8 . & Hydraulic Diameter & $\checkmark$ & $\checkmark$ & $\checkmark$ & $\checkmark$ & $\checkmark$ & $\checkmark$ & $\checkmark$ & $\checkmark$ & $\checkmark$ & $\checkmark$ & $\checkmark$ & $\checkmark$ & $\checkmark$ & $\checkmark$ & $\checkmark$ & $\mathrm{x}$ & $\mathrm{x}$ & $\mathrm{x}$ & $\mathrm{x}$ & $\mathrm{x}$ \\
\hline 2.9 . & Section & $\checkmark$ & $\checkmark$ & $\checkmark$ & $\checkmark$ & $\checkmark$ & $\checkmark$ & $\checkmark$ & $\checkmark$ & $\checkmark$ & $\checkmark$ & $\checkmark$ & $\checkmark$ & $\checkmark$ & $\checkmark$ & $\checkmark$ & $\mathrm{x}$ & $\mathrm{x}$ & $\mathrm{x}$ & $\mathrm{x}$ & $\mathrm{x}$ \\
\hline 2.10 . & Area & $\checkmark$ & $\checkmark$ & $\checkmark$ & $\checkmark$ & $\checkmark$ & $\checkmark$ & $\checkmark$ & $\checkmark$ & $\checkmark$ & $\checkmark$ & $\checkmark$ & $\checkmark$ & $\checkmark$ & $\checkmark$ & $\checkmark$ & $\mathrm{x}$ & $\mathrm{x}$ & $\mathrm{x}$ & $\mathrm{x}$ & $\mathrm{x}$ \\
\hline 3 & Mechanical - Flow & & & & & & & & & & & & & & & & & & & & \\
\hline 3.1 . & Flow & - & - & - & - & - & - & $\checkmark$ & $\checkmark$ & $\checkmark$ & $\checkmark$ & - & - & - & $\checkmark$ & $\checkmark$ & - & - & - & $\mathrm{x}$ & $\mathrm{x}$ \\
\hline 3.2 . & Additional Flow & - & $\begin{array}{ll}- \\
\end{array}$ & - & - & - & \begin{tabular}{|l|}
- \\
\end{tabular} & $\checkmark$ & - & - & $\checkmark$ & - & - & - & $\checkmark$ & $\checkmark$ & - & - & - & - & - \\
\hline 3.3 . & Velocity & - & - & - & - & - & $\begin{array}{ll}- \\
\end{array}$ & $\checkmark$ & $\checkmark$ & - & $\checkmark$ & - & - & - & $\checkmark$ & - & - & - & - & $\mathrm{x}$ & - \\
\hline 3.4. & Friction & - & - & - & - & - & - & $\checkmark$ & - & - & $\checkmark$ & - & - & - & - & - & - & - & - & - & - \\
\hline 3.5 & Pressure Drop & - & - & - & - & - & - & $\checkmark$ & - & - & $\checkmark$ & - & \begin{tabular}{|l|}
- \\
\end{tabular} & - & - & - & - & - & - & - & - \\
\hline 3.6. & Velocity Pressure & - & - & - & - & - & - & $\checkmark$ & - & - & $\checkmark$ & - & - & - & - & - & - & - & - & - & - \\
\hline 3.7. & Reynold Number & - & - & - & - & - & - & $\checkmark$ & - & - & $\checkmark$ & - & - & - & - & - & - & - & - & - & - \\
\hline 3.8. & Roughness & - & - & - & - & - & $\begin{array}{ll}- \\
\end{array}$ & $\checkmark$ & - & - & $\checkmark$ & - & - & - & - & - & - & - & - & - & - \\
\hline 4 & Dimensions & & & & & & & & & & & & & & & & & & & & \\
\hline 4.1. & Size & $\checkmark$ & $\checkmark$ & $\checkmark$ & $\checkmark$ & $\checkmark$ & $\checkmark$ & $\checkmark$ & $\checkmark$ & $\checkmark$ & $\checkmark$ & $\checkmark$ & $\checkmark$ & $\checkmark$ & $\checkmark$ & $\checkmark$ & $\mathrm{x}$ & $\mathrm{x}$ & $\mathrm{x}$ & $\mathrm{x}$ & $\mathrm{x}$ \\
\hline 4.2 . & Width & $\checkmark$ & $\checkmark$ & $\checkmark$ & $\checkmark$ & $\checkmark$ & $\checkmark$ & $\checkmark$ & $\checkmark$ & $\checkmark$ & $\checkmark$ & $\checkmark$ & $\checkmark$ & $\checkmark$ & $\checkmark$ & $\checkmark$ & $\mathrm{x}$ & $\mathrm{x}$ & $\mathrm{x}$ & $\mathrm{x}$ & $\mathrm{x}$ \\
\hline 4.3 . & Height & $\checkmark$ & $\checkmark$ & $\checkmark$ & $\checkmark$ & $\checkmark$ & $\checkmark$ & $\checkmark$ & $\checkmark$ & $\checkmark$ & $\checkmark$ & $\checkmark$ & $\checkmark$ & $\checkmark$ & $\checkmark$ & $\checkmark$ & $\mathrm{x}$ & $\mathrm{x}$ & $\mathrm{x}$ & $\mathrm{x}$ & $\mathrm{x}$ \\
\hline 4.4 . & Length & $\checkmark$ & $\checkmark$ & $\checkmark$ & $\checkmark$ & $\checkmark$ & $\checkmark$ & $\checkmark$ & $\checkmark$ & $\checkmark$ & $\checkmark$ & $\checkmark$ & $\checkmark$ & $\checkmark$ & $\checkmark$ & $\checkmark$ & $\mathrm{x}$ & $\mathrm{x}$ & $\mathrm{x}$ & $\mathrm{x}$ & $\mathrm{x}$ \\
\hline 4.5 . & Diameter & $\checkmark$ & $\checkmark$ & $\checkmark$ & $\checkmark$ & $\checkmark$ & $\checkmark$ & $\checkmark$ & $\checkmark$ & $\checkmark$ & $\checkmark$ & $\checkmark$ & $\checkmark$ & $\checkmark$ & $\checkmark$ & $\checkmark$ & $\mathrm{x}$ & $\mathrm{x}$ & $\mathrm{x}$ & $\mathrm{x}$ & $\mathrm{x}$ \\
\hline 5 & Identity Data & & & & & & & & & & & & & & & & & - & & & \\
\hline 5.1. & Image & - & - & - & - & - & $\begin{array}{ll}- \\
\end{array}$ & - & - & - & $\checkmark$ & - & - & - & - & - & - & - & - & - & - \\
\hline 5.2 . & Comments & - & - & - & - & - & $\begin{array}{ll}- \\
\end{array}$ & $\checkmark$ & - & - & $\checkmark$ & - & $-{ }_{-}^{-}$ & - & $\checkmark$ & - & - & - & - & - & - \\
\hline 5.3. & Mark & - & - & - & - & $\checkmark$ & - & $\checkmark$ & - & $\checkmark$ & $\checkmark$ & - & - & - & $\checkmark$ & - & - & - & - & - & - \\
\hline 5.4. & Keynote & - & - & - & - & - & - & - & - & - & $\checkmark$ & - & - & - & - & - & - & - & - & - & - \\
\hline 5.5 & Model & - & - & - & - & $\checkmark$ & \begin{tabular}{|l|}
- \\
\end{tabular} & $\checkmark$ & - & $\checkmark$ & - & - & - & - & $\checkmark$ & $\checkmark$ & - & - & - & - & - \\
\hline 5.6. & Manufacturer & - & - & - & - & - & $\begin{array}{ll}- \\
\end{array}$ & $\checkmark$ & - & - & $\checkmark$ & - & - & $\checkmark$ & - & $\checkmark$ & - & - & - & - & - \\
\hline 5.7. & URL & - & - & - & - & - & - & - & - & - & $\checkmark$ & - & \begin{tabular}{l|}
- \\
\end{tabular} & - & - & $\checkmark$ & - & - & - & - & - \\
\hline 5.8. & Description & - & - & $\checkmark$ & - & $\checkmark$ & - & - & - & - & $\checkmark$ & - & - & - & $\checkmark$ & - & - & - & - & - & - \\
\hline 5.9. & Assembly Description & - & $\checkmark$ & $\checkmark$ & - & $\checkmark$ & - & $\checkmark$ & - & $\checkmark$ & $\checkmark$ & - & \begin{tabular}{|l|}
- \\
\end{tabular} & - & - & $\checkmark$ & - & - & - & - & $\mathrm{x}$ \\
\hline 5.10. & Assembly Code & - & $\checkmark$ & $\checkmark$ & $\checkmark$ & $\checkmark$ & $\checkmark$ & $\checkmark$ & $\checkmark$ & $\checkmark$ & $\checkmark$ & - & - & - & - & $\checkmark$ & - & - & - & - & $\mathrm{x}$ \\
\hline 5.11. & Cost & - & - & - & - & $\checkmark$ & - & - & - & $\sqrt{ }$ & $\checkmark$ & - & - & $\checkmark$ & - & - & - & - & - & - & - \\
\hline 6 & Insulation & & & & & & & & & & & & & & & & & & & & \\
\hline 6.1 & Overall Size & $\checkmark$ & $\checkmark$ & $\checkmark$ & $\checkmark$ & $\checkmark$ & $\checkmark$ & $\checkmark$ & $\checkmark$ & $\checkmark$ & $\checkmark$ & $\checkmark$ & $\checkmark$ & $\checkmark$ & $\checkmark$ & $\checkmark$ & $\mathrm{x}$ & $\mathrm{x}$ & $\mathrm{x}$ & $\mathrm{x}$ & $\mathrm{x}$ \\
\hline 6.2 . & Insulation Thickness & $\checkmark$ & $\checkmark$ & $\checkmark$ & $\checkmark$ & $\checkmark$ & $\checkmark$ & $\checkmark$ & $\checkmark$ & $\checkmark$ & $\checkmark$ & $\checkmark$ & $\checkmark$ & $\checkmark$ & $\checkmark$ & $\checkmark$ & $\mathrm{x}$ & $\mathrm{x}$ & $\mathrm{x}$ & $\mathrm{x}$ & $\mathrm{x}$ \\
\hline 6.3. & Insulation Type & $\checkmark$ & $\checkmark$ & $\checkmark$ & $\checkmark$ & $\checkmark$ & $\checkmark$ & $\checkmark$ & $\checkmark$ & $\checkmark$ & $\checkmark$ & $\checkmark$ & $\checkmark$ & $\checkmark$ & $\checkmark$ & $\checkmark$ & $\mathrm{x}$ & $\mathrm{x}$ & $\mathrm{x}$ & $\mathrm{x}$ & $\mathrm{x}$ \\
\hline 7 & Lining & & & & & & & & & & & & & & & & & & & & \\
\hline 7.1. & Free Size & $\checkmark$ & $\checkmark$ & $\checkmark$ & $\checkmark$ & $\checkmark$ & $\checkmark$ & $\checkmark$ & $\checkmark$ & $\checkmark$ & $\checkmark$ & $\checkmark$ & $\checkmark$ & $\checkmark$ & $\checkmark$ & $\checkmark$ & $\mathrm{x}$ & $\mathrm{x}$ & $\mathrm{x}$ & $\mathrm{x}$ & $\mathrm{x}$ \\
\hline 7.2 . & Lining Thickness & $\checkmark$ & $\checkmark$ & $\checkmark$ & $\checkmark$ & $\checkmark$ & $\checkmark$ & $\checkmark$ & $\checkmark$ & $\checkmark$ & $\checkmark$ & $\checkmark$ & $\checkmark$ & $\checkmark$ & $\checkmark$ & $\checkmark$ & $\mathrm{x}$ & $\mathrm{x}$ & $\mathrm{x}$ & $\mathrm{x}$ & $\mathrm{x}$ \\
\hline 7.3. & Lining Type & $\checkmark$ & $\checkmark$ & $\checkmark$ & $\checkmark$ & $\checkmark$ & $\checkmark$ & $\checkmark$ & $\checkmark$ & $\checkmark$ & $\checkmark$ & $\checkmark$ & $\checkmark$ & $\checkmark$ & $\checkmark$ & $\checkmark$ & $\mathrm{x}$ & $\mathrm{x}$ & $x$ & $x$ & $\mathrm{x}$ \\
\hline 8 & Accessories & & & & & & & & & & & & & & & & & & & & \\
\hline 8.1 . & Duct placeholder & - & $\checkmark$ & $\checkmark$ & $\checkmark$ & $\checkmark$ & $\checkmark$ & $\checkmark$ & $\checkmark$ & - & $\checkmark$ & - & - & - & - & - & - & - & - & - & - \\
\hline 8.2 . & Duct fitting (many types) & $\checkmark$ & $\checkmark$ & $\checkmark$ & $\checkmark$ & $\checkmark$ & $\checkmark$ & $\checkmark$ & $\checkmark$ & - & $\checkmark$ & $\checkmark$ & $\checkmark$ & $\checkmark$ & $\checkmark$ & $\checkmark$ & $\mathrm{x}$ & $\mathrm{x}$ & $\mathrm{x}$ & $\mathrm{x}$ & - \\
\hline 8.3 . & Damper (many types) & $\checkmark$ & $\checkmark$ & $\checkmark$ & $\checkmark$ & $\checkmark$ & $\checkmark$ & $\checkmark$ & $\checkmark$ & $\checkmark$ & $\checkmark$ & $\checkmark$ & $\checkmark$ & $\checkmark$ & $\checkmark$ & $\checkmark$ & $\mathrm{x}$ & $\mathrm{x}$ & $\mathrm{x}$ & $\mathrm{x}$ & $\mathrm{x}$ \\
\hline 8.4 . & Filter & $\checkmark$ & $\checkmark$ & $\checkmark$ & $\checkmark$ & $\checkmark$ & $\checkmark$ & $\checkmark$ & $\checkmark$ & $\checkmark$ & $\checkmark$ & $\checkmark$ & $\checkmark$ & $\checkmark$ & $\checkmark$ & $\checkmark$ & $\mathrm{x}$ & $\mathrm{x}$ & $\mathrm{x}$ & $\mathrm{x}$ & $\mathrm{x}$ \\
\hline 8.5 . & Air terminal & $\checkmark$ & $\checkmark$ & $\checkmark$ & $\checkmark$ & $\checkmark$ & $\checkmark$ & $\checkmark$ & $\checkmark$ & $\checkmark$ & $\checkmark$ & $\checkmark$ & $\checkmark$ & $\checkmark$ & $\checkmark$ & $\checkmark$ & $\mathrm{x}$ & $\mathrm{x}$ & $\mathrm{x}$ & $\mathrm{x}$ & $\mathrm{x}$ \\
\hline 8.6. & Fitting (many types) & $\checkmark$ & $\checkmark$ & $\checkmark$ & $\checkmark$ & $\checkmark$ & $\checkmark$ & $\checkmark$ & $\checkmark$ & - & $\checkmark$ & $\checkmark$ & $\checkmark$ & $\checkmark$ & $\checkmark$ & $\checkmark$ & $\mathrm{x}$ & $\mathrm{x}$ & $\mathrm{x}$ & $\mathrm{x}$ & - \\
\hline 9 & Additional Information & & & & & & & & & & & & & & & & & & & & \\
\hline 9.1. & WBS Code & $\checkmark$ & $\checkmark$ & - & $\checkmark$ & - & $\checkmark$ & - & - & $\checkmark$ & $\checkmark$ & - & $\checkmark$ & - & - & - & - & - & - & - & - \\
\hline 9.2. & BOQ Code & $\checkmark$ & - & - & - & $\checkmark$ & $\checkmark$ & - & - & $\checkmark$ & $\checkmark$ & - & - & $\checkmark$ & - & \begin{tabular}{|l}
- \\
\end{tabular} & - & - & $\mathrm{x}$ & - & - \\
\hline 9.3. & Installation Date & - & $\checkmark$ & $\checkmark$ & $\checkmark$ & - & - & - & - & $\checkmark$ & $\checkmark$ & - & - & - & - & $\checkmark$ & - & - & - & - & $\mathrm{x}$ \\
\hline 9.4. & Commission date & - & - & $\checkmark$ & $\checkmark$ & - & - & - & - & $\checkmark$ & $\checkmark$ & - & - & - & - & $\checkmark$ & - & - & - & - & $\mathrm{x}$ \\
\hline 9.5. & Warranty period. & - & - & $\checkmark$ & - & - & - & - & - & $\checkmark$ & $\checkmark$ & - & - & - & - & $\checkmark$ & - & - & - & - & $\mathrm{x}$ \\
\hline \begin{tabular}{|l|l}
58 \\
\end{tabular} & & & & & & & & & & & & & & & & & 32 & 32 & 33 & 34 & 36 \\
\hline
\end{tabular}

2-3 respondents of both G1\&G2 agreed but not $\mathrm{G} 3$

2-3 respondents of $\mathrm{G} 3$ agreed but no $2-3$ selections by both $\mathrm{G} 1 \& \mathrm{G} 2$ 
Table (4): Frequently selected properties by groups G1, G2 and G3 for pipes.

\begin{tabular}{|c|c|c|c|c|c|c|c|c|c|c|c|c|c|c|c|c|c|c|c|c|c|}
\hline \multirow{2}{*}{\multicolumn{2}{|c|}{\begin{tabular}{l|l}
\multicolumn{2}{c}{ Properties } \\
$\#$ & Pipe Item \\
$\#$
\end{tabular}}} & \multicolumn{10}{|c|}{$\begin{array}{l}\text { Mutual 2-3 answers to be considered by } \\
\mathrm{G} 1+\mathrm{G} 2\end{array}$} & \multirow{2}{*}{\multicolumn{5}{|c|}{$\begin{array}{l}\text { Mutual 2-3 answers to be } \\
\text { considered by G3 } \\
\text { Clsh 4D } \text { QTO ShD FM }\end{array}$}} & \multicolumn{5}{|c|}{\begin{tabular}{|l|}
$\begin{array}{l}\text { Required information } \\
\text { items }(\mathrm{G} 1+\mathrm{G} 2)+\mathrm{G} 3\end{array}$ \\
\end{tabular}} \\
\hline & & $\overline{\mathrm{Cl}}$ & sh & $\overline{4 \mathrm{I}}$ & & $\overline{Q T}$ & $\mathrm{TO}$ & $\mathrm{Sh}$ & & $\overline{F N}$ & & & & & & & Clsh & $4 \mathrm{D}$ & $\overline{\mathrm{QTO}}$ & $\mathrm{ShD}$ & \\
\hline & Pipe Type & G1 & \begin{tabular}{|l|l|}
$\mathrm{G} 2$ & \\
\end{tabular} & G1 & $\mathrm{G} 2$ & G1 & $\mathrm{G} 2$ & G1 & $\mathrm{G} 2$ & G1 & & & $2 / 3=$ & $3 / 3=$ & $=" \sqrt{ }=$ & & If (G1 & $1 \& 0$ & i2) \& & $\mathrm{G} 3=$ & \\
\hline 1 & Constraints & & $(2 / 38)$ & $\begin{array}{l}\& 2 / 3) \\
=(0 / 3\end{array}$ & $\begin{array}{l}=(2 / 3 \\
3 \& 0 / 3\end{array}$ & $\begin{array}{l}3 \& 3 / 3 \\
3)=(1\end{array}$ & $\begin{array}{l}3)=(3 \\
13 \& 1\end{array}$ & $13 \& 3 /$ & $\begin{array}{l}3)= \\
1 / 3 \&\end{array}$ & 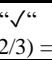 & & & $1 / 3=$ & $=0 / 3=$ & =“"“ & & then 1 & $\begin{array}{l}\text { prope } \\
\text { othel }\end{array}$ & $\begin{array}{l}\text { ty ob } \\
\text { wise }\end{array}$ & $\begin{array}{l}\text { ains an } \\
=" “ " “\end{array}$ & \\
\hline 1.1. & Horizontal Justification & $\begin{array}{ll}\checkmark \\
\end{array}$ & \begin{tabular}{|l|l} 
& \\
\end{tabular} & $\checkmark$ & $\checkmark$ & $\checkmark$ & \begin{tabular}{|l|l|l|l|}
$\checkmark$ \\
\end{tabular} & $\checkmark \mid$ & \begin{tabular}{|l|l|l|}
$\checkmark$ & -1
\end{tabular} & $\sqrt{ }$ & $\sqrt{ }$ & $\sqrt{ }$ & $\sqrt{v}$ & 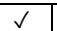 & $\bar{v}$ & $\checkmark$ & $\mathrm{x}$ & 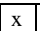 & $\mathrm{x}$ & $\mathrm{x}$ & \\
\hline 1.2 . & Vertical Justification & $\checkmark$ & $\checkmark \mid$ & $\checkmark$ & $\checkmark$ & $\checkmark$ & $\checkmark$ & $\checkmark$ & $\checkmark$ & $\checkmark$ & $\checkmark$ & $\checkmark$ & $\checkmark$ & $\checkmark$ & $\checkmark$ & $\checkmark$ & $\mathrm{x}$ & $\mathrm{x}$ & $\mathrm{x}$ & $\mathrm{x}$ & $\mathrm{x}$ \\
\hline 1.3 . & Reference level & $\checkmark$ & $\checkmark \mid$ & $\checkmark$ & $\checkmark$ & $\checkmark$ & $\checkmark$ & $\checkmark$ & $\checkmark$ & $\checkmark$ & $\checkmark$ & $\checkmark$ & $\checkmark$ & $\checkmark$ & $\checkmark$ & $\checkmark$ & $\mathrm{x}$ & $\mathrm{x}$ & $\mathrm{x}$ & $\mathrm{x}$ & $\mathrm{x}$ \\
\hline 1.4 . & Start Offset & $\checkmark$ & $\checkmark$ & $\checkmark$ & $\checkmark$ & $\checkmark$ & $\checkmark$ & $\checkmark$ & $\checkmark$ & $\begin{array}{ll} \\
\end{array}$ & $\checkmark$ & $\checkmark$ & $\sqrt{ }$ & $\checkmark$ & $\checkmark$ & $\checkmark$ & $\mathrm{x}$ & $\mathrm{x}$ & $\mathrm{x}$ & $\mathrm{x}$ & $\mathrm{x}$ \\
\hline 1.5 . & End Offset & \begin{tabular}{|lll} 
\\
\end{tabular} & \begin{tabular}{|l|l}
$\checkmark$ & \\
\end{tabular} & $\checkmark$ & $\checkmark$ & $\checkmark$ & \begin{tabular}{|lll}
$\checkmark$ & & \\
\end{tabular} & 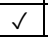 & \begin{tabular}{|l|l|l|l}
$\checkmark$ & \\
\end{tabular} & $\mathrm{v}$ & $\checkmark$ & $\checkmark$ & $\sqrt{ }$ & $\checkmark$ & $\checkmark$ & $\checkmark$ & $\mathrm{x}$ & $\mathrm{x}$ & $\mathrm{x}$ & $\mathrm{x}$ & $\mathrm{x}$ \\
\hline 1.6 . & Slope & $\checkmark$ & 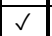 & $\checkmark$ & $\checkmark$ & \begin{tabular}{|l|l|l}
$\checkmark$ & \\
\end{tabular} & $\checkmark$ & \begin{tabular}{|l|l}
$\checkmark$ & \\
\end{tabular} & $\checkmark$ & $a^{\prime}$ & $\checkmark$ & $\checkmark$ & $\checkmark$ & $\checkmark$ & $\checkmark$ & $\checkmark$ & \begin{tabular}{l|l}
$x$ \\
\end{tabular} & $\mathrm{x}$ & $\mathrm{x}$ & $\mathrm{x}$ & $\mathrm{x}$ \\
\hline 2 & Mechanical & & & & & & & & & & & & & & & & & & & & \\
\hline 2.1 . & \begin{tabular}{|l|} 
System Classification \\
\end{tabular} & $\checkmark$ & \begin{tabular}{|l|ll} 
& \\
\end{tabular} & $\checkmark$ & $\checkmark$ & $\checkmark$ & $\checkmark$ & 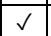 & $\checkmark$ & $\mathrm{s}$ & $\checkmark$ & $\checkmark$ & $\checkmark$ & $\checkmark$ & $\checkmark$ & $\checkmark$ & $\mathrm{x}$ & $\mathrm{x}$ & $\mathrm{x}$ & $\mathrm{x}$ & $\mathrm{x}$ \\
\hline 2.2 . & System Type & $\begin{array}{llllllll}\checkmark & & l & l & l \\
\end{array}$ & \begin{tabular}{|l|lllll} 
& \\
\end{tabular} & $\checkmark$ & $\begin{array}{llllll} & & & l & l\end{array}$ & \begin{tabular}{|l|l|l|l}
$\checkmark$ \\
\end{tabular} & \begin{tabular}{|l|ll}
$\checkmark$ & & \\
\end{tabular} & $\begin{array}{llllllllll} & \\
\end{array}$ & \begin{tabular}{|l|ll}
$\checkmark$ & & $l$
\end{tabular} & $\checkmark$ & $\checkmark$ & $\checkmark$ & $\sqrt{ }$ & $\checkmark$ & $\checkmark$ & $\checkmark$ & $\mathrm{x}$ & $\mathrm{x}$ & $\mathrm{x}$ & $\mathrm{x}$ & $\mathrm{x}$ \\
\hline 2.3 . & System Name & $\checkmark$ & \begin{tabular}{|l|ll}
$\checkmark$ & &
\end{tabular} & $\checkmark$ & $\checkmark$ & $\checkmark$ & $\checkmark$ & 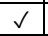 & $\checkmark$ & $\checkmark$ & $\checkmark$ & $\checkmark$ & $\sqrt{ }$ & $\checkmark$ & $\checkmark$ & $\checkmark$ & $\mathrm{x}$ & $\mathrm{x}$ & $\mathrm{x}$ & $\mathrm{x}$ & $\mathrm{x}$ \\
\hline 2.4 . & System Abbreviation & $\checkmark$ & \begin{tabular}{|l|l} 
& \\
\end{tabular} & $\checkmark$ & $\checkmark$ & $\checkmark$ & \begin{tabular}{|l|ll}
$\checkmark$ & & $l$
\end{tabular} & \begin{tabular}{|l|l}
$\checkmark$ & \\
\end{tabular} & \begin{tabular}{|l|l|l|l|l} 
\\
\end{tabular} & $\mathrm{s}$ & $\checkmark$ & $\checkmark$ & $\checkmark$ & 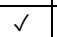 & $\checkmark$ & $\checkmark$ & $\mathrm{x}$ & $\mathrm{x}$ & $\mathrm{x}$ & $\mathrm{x}$ & $\mathrm{x}$ \\
\hline 2.5 . & Pipe segment $($ steel,$\ldots)$ & $\checkmark$ & $\checkmark \mid$ & $\checkmark$ & $\checkmark$ & $\checkmark$ & $\checkmark$ & $\checkmark \mid$ & $\checkmark$ & $\checkmark$ & $\checkmark$ & $\checkmark$ & $\checkmark$ & $\checkmark$ & $\checkmark$ & $\checkmark$ & $\mathrm{x}$ & $\mathrm{x}$ & $\mathrm{x}$ & $\mathrm{x}$ & $\mathrm{x}$ \\
\hline 2.6 . & connection type & $\checkmark$ & \begin{tabular}{|l|ll} 
& &
\end{tabular} & $\checkmark$ & $\checkmark$ & \begin{tabular}{|l|l|l}
$\checkmark$ \\
\end{tabular} & \begin{tabular}{|l|ll}
$\checkmark$ & & $l$
\end{tabular} & 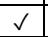 & \begin{tabular}{|l|ll}
$\checkmark$ & & $l$
\end{tabular} & $\mathrm{~s}$ & $\checkmark$ & $\checkmark$ & $\checkmark$ & $\checkmark$ & $\sqrt{ }$ & $\checkmark$ & $\mathrm{x}$ & $\mathrm{x}$ & $\mathrm{x}$ & $\mathrm{x}$ & $\mathrm{x}$ \\
\hline 2.7 . & Roughness & - & - & - & - & - & - & - & $\checkmark$ & - & $\checkmark$ & - & - & - & - & - & - & -1 & - & - & - \\
\hline 2.8 . & Material & - & 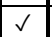 & - & - & - & \begin{tabular}{|l|l|l|l}
$\checkmark$ & \\
\end{tabular} & \begin{tabular}{|l|l|l|l|l}
$\checkmark$ & \\
\end{tabular} & \begin{tabular}{|l|l|l|l} 
\\
\end{tabular} & - & $\checkmark$ & - & - & $\sqrt{2}$ & $\checkmark$ & - & - & - & - & $\mathrm{x}$ & - \\
\hline 2.9 . & Schedule / type & \begin{tabular}{|l}
- \\
\end{tabular} & \begin{tabular}{|l|l|}
$\checkmark$ & \\
\end{tabular} & $\checkmark$ & - & $\checkmark$ & $\checkmark$ & $\checkmark \mid$ & - & - & $\checkmark$ & - & - & $\checkmark$ & - & - & - & - & $\mathrm{x}$ & - & - \\
\hline 2.10 . & Segment description & - & - & - & - & - & $\checkmark$ & $\checkmark$ & - & - & $\checkmark$ & - & - & - & - & - & - & - & - & - & - \\
\hline 2.11 . & Invert elevation & $\checkmark$ & $\checkmark \mid$ & $\checkmark$ & $\checkmark$ & $\checkmark$ & $\checkmark$ & $\checkmark$ & $\checkmark$ & $\checkmark$ & $\checkmark$ & $\checkmark$ & $\checkmark$ & 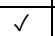 & $\checkmark$ & $\checkmark$ & $\mathrm{x}$ & $\mathrm{x}$ & $\mathrm{x}$ & $\mathrm{x}$ & $\mathrm{x}$ \\
\hline 2.12 . & Section & $\checkmark$ & $\checkmark$ & $\checkmark$ & $\checkmark$ & \begin{tabular}{|l|l|l}
$\checkmark$ & \\
\end{tabular} & $\checkmark$ & 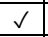 & $\checkmark$ & $\checkmark$ & $\sqrt{ }$ & $\checkmark$ & $\checkmark$ & $\checkmark$ & $\sqrt{ }$ & $\checkmark$ & $\mathrm{x}$ & $\mathrm{x}$ & $\mathrm{x}$ & $\mathrm{x}$ & $\mathrm{x}$ \\
\hline 2.13 . & Area & $\checkmark$ & 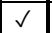 & $\checkmark$ & $\checkmark$ & $\checkmark$ & $\checkmark \mid$ & $\checkmark \mid$ & $\checkmark$ & $\checkmark$ & $\checkmark$ & $\checkmark$ & $\sqrt{ }$ & $\checkmark$ & $\checkmark$ & $\checkmark$ & \begin{tabular}{l|l}
$x$ \\
\end{tabular} & $\mathrm{x}$ & $\mathrm{x}$ & $\mathrm{x}$ & $\mathrm{x}$ \\
\hline 3 & Mechanical - Flow & & 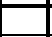 & & 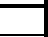 & & & & & & & & 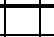 & & & 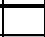 & & & & & \\
\hline 3.1 . & \begin{tabular}{|l|} 
Flow \\
\end{tabular} & - & - & - & - & -- & $-{ }_{-1}$ & $\checkmark$ & $\checkmark$ & - & $\checkmark$ & - & $\mid-$ & - & $\checkmark$ & $\checkmark$ & - & $-\left.\right|_{-1}$ & - & $\mathrm{x}$ & - \\
\hline 3.2 . & Additional Flow & - & - & - & - & - & - & \begin{tabular}{|l|l|l|l} 
\\
\end{tabular} & - & - & $\checkmark$ & - & - & - & $\checkmark$ & $\checkmark$ & - & -1 & - & - & - \\
\hline 3.3 . & Velocity & - & - & - & - & $-{ }_{-1}$ & - & \begin{tabular}{|l|l|l}
$\checkmark$ & \\
\end{tabular} & $\checkmark$ & - & $\checkmark$ & - & - & - & $\checkmark$ & - & - & - & - & $\mathrm{x}$ & - \\
\hline 3.4. & Friction & $\begin{array}{ll}- \\
\end{array}$ & - & - & - & \begin{tabular}{|l|}
- \\
\end{tabular} & - & \begin{tabular}{|l|l} 
\\
\end{tabular} & $\begin{array}{ll}- \\
\end{array}$ & - & $\checkmark$ & - & - & - & - & - & - & - & - & - & - \\
\hline 3.5 . & Friction factor & - & - & - & - & - & - & - & - & - & $\checkmark$ & - & - & - & - & - & - & - & - & - & - \\
\hline 3.6 . & Relative roughness & -- & - & - & - & - & - & - & - & - & $\checkmark$ & - & - & - & - & - & - & - & - & - & - \\
\hline 3.7. & Flow state & \begin{tabular}{|l}
- \\
\end{tabular} & - & - & - & - & - & - & - & - & $\checkmark$ & - & - & - & - & - & - & - & - & - & - \\
\hline 3.8 . & Reynold Number & - & - & - & - & - & \begin{tabular}{|l|l|}
- & \\
\end{tabular} & - & $\begin{array}{ll}- \\
\end{array}$ & - & $\checkmark$ & - & - & - & - & - & - & - & - & - & - \\
\hline 4 & Dimensions & & 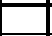 & & 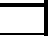 & & & & & & & & - & & & 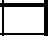 & + & & & & 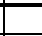 \\
\hline 4.1. & Size & $\checkmark$ & \begin{tabular}{|l|l|}
$\checkmark$ & \\
\end{tabular} & $\checkmark$ & $\checkmark \mid$ & $\checkmark$ & \begin{tabular}{|l|l|}
$\checkmark$ \\
\end{tabular} & \begin{tabular}{|l|l} 
\\
\end{tabular} & \begin{tabular}{|l|l|}
$\checkmark$ \\
\end{tabular} & $\begin{array}{ll} \\
\end{array}$ & $\checkmark$ & $\checkmark$ & $\sqrt{ }$ & $\checkmark$ & $\checkmark$ & $\checkmark$ & $\mathrm{x}$ & $\mathrm{x}$ & $\mathrm{x}$ & $\mathrm{x}$ & $\mathrm{x}$ \\
\hline 4.2 . & Outside diameter & $\checkmark$ & $\checkmark$ & $\checkmark$ & $\checkmark$ & $\checkmark$ & $\checkmark$ & \begin{tabular}{|l|l|}
$\checkmark$ & \\
\end{tabular} & $\checkmark$ & $\checkmark$ & \begin{tabular}{|l|l|} 
& \\
\end{tabular} & $\checkmark$ & $\checkmark$ & $\checkmark$ & $\checkmark$ & $\checkmark$ & \begin{tabular}{l|l|}
$\mathrm{x}$ & $\mathrm{y}$ \\
\end{tabular} & \begin{tabular}{|l|l|}
$x$ & \\
\end{tabular} & $\mathrm{x}$ & $\mathrm{x}$ & $\mathrm{x}$ \\
\hline 4.3 . & Inside diameter & $\checkmark$ & $\checkmark$ & $\checkmark$ & $\checkmark \mid$ & \begin{tabular}{|l|l|}
$\checkmark$ \\
\end{tabular} & \begin{tabular}{|l|l|}
$\checkmark$ \\
\end{tabular} & \begin{tabular}{|l|l|} 
& \\
\end{tabular} & \begin{tabular}{|l|l|} 
\\
\end{tabular} & $\checkmark$ & $\checkmark \mid$ & $\checkmark$ & $\sqrt{2}$ & $\checkmark$ & $\checkmark$ & $\checkmark$ & \begin{tabular}{l|l}
$x$ \\
\end{tabular} & $\mathrm{x}$ & $\mathrm{x}$ & $\mathrm{x}$ & $\mathrm{x}$ \\
\hline 4.4 . & Length & $\checkmark$ & $\checkmark$ & $\checkmark$ & $\checkmark$ & \begin{tabular}{|l|}
$\checkmark$ \\
\end{tabular} & $\checkmark$ & \begin{tabular}{|l|l} 
\\
\end{tabular} & $\checkmark$ & $\begin{array}{llll} & \end{array}$ & \begin{tabular}{|l|l|}
$\checkmark$ & \\
\end{tabular} & $\checkmark$ & $\checkmark$ & $\checkmark$ & $\checkmark$ & $\checkmark$ & \begin{tabular}{l|l}
$x$ \\
\end{tabular} & $\mathrm{x}$ & $\mathrm{x}$ & $\mathrm{x}$ & $\mathrm{x}$ \\
\hline 5 & Identity Data & & & & & & & & & & & & & & & & & & & & \\
\hline 5.1 . & Image & $\begin{array}{ll}- \\
\end{array}$ & - & - & - & - & - & -1 & - & $\sqrt{ }$ & \begin{tabular}{|l|l|l|l|l|l|l|l} 
&
\end{tabular} & - & - & - & - & - & - & - & - & - & $-{ }_{-1}$ \\
\hline 5.2 . & Comments & - & - & - & - & - & - & - & - & - & $\checkmark$ & - & - & - & $\checkmark$ & - & - & - & - & - & - \\
\hline 5.3 . & Mark & - & - & - & - & - & - & $\checkmark \mid$ & - & $\checkmark$ & $\checkmark$ & - & - & - & $\checkmark$ & - & - & -1 & - & - & - \\
\hline 5.4 . & Keynote & - & - & $\checkmark$ & - & - & - & $\checkmark$ & - & - & $\checkmark$ & - & - & - & - & - & - & - & - & - & - \\
\hline 5.5 . & Model & - & - & - & - & $\checkmark$ & - & - & - & $\checkmark$ & - & - & - & - & $\checkmark$ & $\checkmark$ & - & $-\left.\right|_{-1}$ & - & - & - \\
\hline 5.6 . & Manufacturer & - & - & - & - & - & - & - & - & - & $\checkmark$ & - & - & 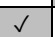 & - & $\checkmark$ & - & -1 & - & - & - \\
\hline 5.7 . & URL & - & -- & - & - & $-{ }_{-1}$ & - & - & - & - & $\sqrt{ }$ & - & - & - & - & $\checkmark$ & - & - & - & - & - \\
\hline 5.8 . & Description & \begin{tabular}{|l}
- \\
\end{tabular} & - & - & - & $\begin{array}{ll}- \\
\end{array}$ & - & \begin{tabular}{|l|l}
$\checkmark$ & \\
\end{tabular} & - & - & 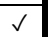 & - & - & - & $\checkmark$ & - & - & - & - & - & - \\
\hline 5.9 . & Assembly Description & - & $\checkmark$ & $\checkmark$ & - & - & - & \begin{tabular}{|l|l}
$\checkmark$ & \\
\end{tabular} & \begin{tabular}{|l|l|l|l|l} 
\\
\end{tabular} & 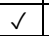 & $\checkmark$ & - & - & - & - & $\checkmark$ & - & - & - & - & $\mathrm{x}$ \\
\hline 5.10 . & Assembly Code & - & $\checkmark$ & $\checkmark$ & $\checkmark$ & - & $\checkmark$ & $\checkmark$ & $\checkmark$ & $\checkmark$ & $\checkmark$ & - & - & - & - & $\checkmark$ & - & - & - & - & $\mathrm{x}$ \\
\hline 5.11 . & Cost & \begin{tabular}{|l}
- \\
\end{tabular} & - & - & - & $\checkmark \mid$ & - & - & - & - & \begin{tabular}{|l|lll}
$\checkmark$ & & & \\
\end{tabular} & - & - & - & - & - & - & - & - & - & - \\
\hline 6 & Insulation & & & & & & & & & & & & & & & & & & & & \\
\hline 6.1 . & Overall Size & $\checkmark$ & $\checkmark$ & $\checkmark$ & $\checkmark$ & $\checkmark$ & $\checkmark$ & $\checkmark$ & $\checkmark$ & $\checkmark$ & $\checkmark$ & $\checkmark$ & I & 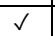 & $\checkmark$ & $\checkmark$ & $\mathrm{x}$ & $\mathrm{x}$ & $\mathrm{x}$ & $\mathrm{x}$ & $\mathrm{x}$ \\
\hline 6.2 . & Insulation Thickness & $\checkmark$ & $\checkmark$ & $\checkmark$ & $\checkmark$ & $\checkmark$ & $\checkmark$ & \begin{tabular}{|l|l} 
\\
\end{tabular} & $\checkmark$ & $\checkmark$ & \begin{tabular}{|l|l|}
$\checkmark$ & \\
\end{tabular} & $\checkmark$ & $\checkmark$ & $\checkmark$ & $\checkmark$ & $\checkmark$ & $\mathrm{x}$ & $\mathrm{x}$ & $\mathrm{x}$ & $\mathrm{x}$ & $\mathrm{x}$ \\
\hline 6.3 . & Insulation Type & $\checkmark$ & $\checkmark$ & $\checkmark$ & $\checkmark \mid$ & $\checkmark$ & \begin{tabular}{|l|l|} 
\\
\end{tabular} & \begin{tabular}{|l|l}
$\checkmark$ & \\
\end{tabular} & $\checkmark \mid$ & $\checkmark$ & \begin{tabular}{|l|l} 
\\
\end{tabular} & $\checkmark$ & $\sqrt{2}$ & $\checkmark$ & $\checkmark$ & $\checkmark$ & \begin{tabular}{l|l|}
$x$ & \\
\end{tabular} & \begin{tabular}{|l|}
$x$ \\
\end{tabular} & $\mathrm{x}$ & $\mathrm{x}$ & $\mathrm{x}$ \\
\hline 6.4 . & Cost & - & - & - & - & $\checkmark$ & $-{ }^{-}$ & \begin{tabular}{l|l}
- & \\
\end{tabular} & - & - & \begin{tabular}{|l|l|} 
& \\
\end{tabular} & - & - & - & - & - & $-{ }_{-1}$ & - & - & - & - \\
\hline 7 & Accessories & & & & & & & & & & & & & & & & & & & & \\
\hline 7.1 . & $\begin{array}{l}\text { Valve } \\
\end{array}$ & $\checkmark$ & $\checkmark$ & $\checkmark$ & $\checkmark$ & \begin{tabular}{|l|l|}
$\checkmark$ \\
\end{tabular} & $\checkmark \mid$ & \begin{tabular}{|l|l|} 
\\
\end{tabular} & $\checkmark \mid$ & $\checkmark$ & $\checkmark$ & $\checkmark$ & $\checkmark$ & $\checkmark v$ & $\checkmark$ & $\checkmark$ & \begin{tabular}{l|l|}
$x$ & \\
\end{tabular} & $\mathrm{x}$ & $\mathrm{x}$ & $\mathrm{x}$ & $\mathrm{x}$ \\
\hline 7.2 . & Gauge & $\checkmark$ & $\checkmark$ & $\checkmark$ & $\checkmark$ & \begin{tabular}{|l|l|}
$\checkmark$ \\
\end{tabular} & \begin{tabular}{|l|l|}
$\checkmark$ \\
\end{tabular} & \begin{tabular}{|l|l}
$\checkmark$ & \\
\end{tabular} & \begin{tabular}{|l|l|}
$\checkmark$ \\
\end{tabular} & $\checkmark$ & \begin{tabular}{|l|l|}
$\checkmark$ & \\
\end{tabular} & $\checkmark$ & $\checkmark$ & $\checkmark$ & $\checkmark$ & $\checkmark$ & \begin{tabular}{l|l}
$x$ \\
\end{tabular} & $\mathrm{x}$ & $\mathrm{x}$ & $\mathrm{x}$ & $\mathrm{x}$ \\
\hline 7.3 . & Strainer & $\checkmark$ & $\checkmark$ & $\checkmark$ & $\checkmark$ & \begin{tabular}{|l|l}
$\checkmark$ \\
\end{tabular} & $\checkmark$ & \begin{tabular}{|l|l|}
$\checkmark$ & \\
\end{tabular} & $\checkmark$ & $\checkmark$ & \begin{tabular}{|l|l|} 
& \\
\end{tabular} & $\checkmark$ & $\checkmark$ & $\checkmark$ & $\checkmark$ & $\checkmark$ & \begin{tabular}{l|l}
$x$ \\
\end{tabular} & $\mathrm{x}$ & $\mathrm{x}$ & $\mathrm{x}$ & $\mathrm{x}$ \\
\hline 7.4 . & Air Separator & $\checkmark$ & $\checkmark$ & $\checkmark$ & $\checkmark$ & $\checkmark$ & \begin{tabular}{|l|l|} 
\\
\end{tabular} & \begin{tabular}{|l|l|l|} 
& \\
\end{tabular} & \begin{tabular}{|l|l|l} 
\\
\end{tabular} & $\checkmark$ & $\checkmark$ & $\checkmark$ & $\checkmark$ & $\checkmark$ & $\checkmark$ & $\checkmark$ & \begin{tabular}{l|l}
$x$ \\
\end{tabular} & $\mathrm{x}$ & $\mathrm{x}$ & $\mathrm{x}$ & $\mathrm{x}$ \\
\hline 7.5 . & Cost & - & - & - & - & $\checkmark$ & - & $-{ }_{-1}$ & - & $\checkmark$ & $\checkmark$ & - & $\mid-$ & - & - & - & - & - & - & - & \\
\hline 8 & Additional Information & & & & & & & & & & & & & & & & & & & & \\
\hline 8.1 . & WBS Code & - & $\checkmark$ & - & $\checkmark$ & - & $\checkmark$ & - & - & $\bar{v}$ & $\sqrt{ }$ & - & $\checkmark$ & - & - & - & - & - & - & - & \\
\hline 8.2 . & BOQ Code & - & - & - & - & $\checkmark$ & $\checkmark$ & - & - & $\checkmark$ & $\checkmark$ & - & $\mid-$ & $\checkmark$ & - & - & - & - & $\mathrm{x}$ & - & - \\
\hline 8.3 . & Installation Date & - & $\checkmark$ & $\checkmark$ & $\checkmark$ & -- & - & - & - & $\checkmark$ & $\checkmark$ & - & - & - & - & $\checkmark$ & - & & - & - & $\mathrm{x}$ \\
\hline 8.4 . & Commission date & - & -1 & $|\checkmark|$ & $\checkmark$ & -1 & \begin{tabular}{|l|l}
- & \\
\end{tabular} & \begin{tabular}{|l|}
- \\
\end{tabular} & \begin{tabular}{|l|l}
- & \\
\end{tabular} & $\bar{l}$ & 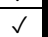 & - & - & - & - & $\checkmark$ & - & - & - & - & $\mathrm{x}$ \\
\hline 8.5 . & Warranty period. & -1 & - & $\checkmark$ & - & - & - & - & - & 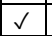 & \begin{tabular}{|l|l}
$\checkmark$ & \\
\end{tabular} & - & - & - & - & $\checkmark$ & - & -1 & - & - & $\mathrm{x}$ \\
\hline 56 & & & & & & & & & & & & & & & & & & 26 & 28 & 29 & 31 \\
\hline
\end{tabular}


If a property was selected by at least two practitioners in all the groups (i.e. shown with " $\checkmark$ " in Tables $3 \& 4$ ), this property was identified as the most frequently selected property for the indicated BIM use in different project types and it was shown with an " $x$ " in the last column group. If a property was selected by at least two respondents in both G1 and G2, but selected by one or no respondent in G3, this property was identified as a property that is only required in transportation/infrastructure projects. This is shown with "_." and shaded dark in Tables 3 and 4 . In the opposite case, if a property was selected by at least two respondents in G3, but selected by one or no respondent in G2 or G3, the related property was relevant for practitioners working in residential/commercial projects and it is shown with "_." and shaded light. Finally, if none or one of the respondents in each group selected a specific property, this property was considered a second-order (i.e., optional) property for each type of the corresponding project type (e.g. duct placeholder for Clsh) and it is shown by “_." in Tables $3 \& 4$.

For ducts, 32 to 36 properties were the most frequently selected properties for the five BIM uses as shown in Table 3. The properties under the categories of "Constraints, Mechanical, Dimensions, Insulation, Lining, and Accessories" were selected by all three groups of respondents except two properties "loss coefficient" in Mechanical Properties, and "duct place holder" in Accessories group of properties. For pipes, the most frequently selected properties varied from 26 to 31 for five BIM uses (Table 4). "Constraints" and "Mechanical" categories were selected by all three groups of respondents for all the defined BIM uses except the following properties in the Mechanical category: "roughness", "material", "schedule/type", and "segment description". In addition, the categories of Dimensions, Insulation and Accessories were selected by all groups, except the "cost" property in both Insulation and Accessories categories. In the interviews, some of the respondents expressed their reluctance to include cost data in the models due to privacy issues or highlighted unavailability of this information while preparing the BIM model.

Some BIM uses required some additional properties compared to the other BIM uses. For example, "BOQ code" in the Additional Information group of properties was required for performing QTO. Another example is the need for "flow" and "velocity" information from the Mechanical-Flow category to perform ShD task. Finally, "flow" property (in the duct case), "Assembly description", "Assembly code", "installation date", "commission date" and "warranty period" were additional requirements to perform FM. The findings corroborate with the literature indicating that FM is a more demanding task for non-geometric information related to post-construction activities such as maintenance and repair works.

The information items that were frequently selected by practitioners in Group 1 and Group 2, and not by Group 3 represent the information items that are important in transportation/infrastructure projects, but not very relevant in residential/commercial projects. These information items include "Work Breakdown Structure (WBS) code" selected for Clsh and FM for ducts, the "BOQ code" property selected for FM in duct and pipes cases, "Installation date" and "commission date" for 4D use for both duct and pipe. These additional properties are probably selected in the transportation/infrastructure projects for managing more complex MEP systems in these types of projects.

The properties that were frequently selected by practitioners in Group 3 and not by Group 1 and Group 2 are represented with light shading in Tables 3 and 4 . These are the properties that are important in residential/commercial projects, but not very relevant in transportation/infrastructure projects. For example, "manufacturer" property was required for QTO and "comment", "mark", "model' and "description" were required for ShD in addition to the "additional flow" property. Finally, for FM, "additional flow", "model", "manufacturer" and "URL" and "fittings" properties were additionally required. This addresses the change in the required information based on the characteristics of each project type according to the pre-defined BIM uses.

Finally, the properties that were not frequently selected by any of the groups were represented by “_“ in the last group of columns of Tables 3 and 4 without any shadings. The Mechanical-Flow category of properties for ducts and pipes were considered as a second priority group of properties in general, probably due to being used specifically in mechanical analyses. The category of Identity Data was also considered a second priority group of properties for duct and pipe components as the related properties received a mixed pattern of selections by practitioners. The Identity Group of properties is mainly not relevant for Clsh and 4D and it is selected in QTO, $\mathrm{ShD}$, and FM. Finally, in both duct and pipe cases, the properties under Additional Information category received less importance by practitioners in all project types, specifically in Clsh, QTO, and ShD except "BOQ code", but showed more importance for 4D and FM uses. These properties are the items required by respondents but not in the original lists of Revit. This emphasizes that additional properties and information can be observed in specific 
project types or based on specific requirements. These were defined also based on the cumulative experience of the involved practitioners, and they need to be evaluated for similar projects in the future.

The findings emphasize how the variation in BIM use determines which information items are to be included in the model and thus affect the modeling effort. It should be noticed that $\mathrm{ShD}$ and FM required more information due to the characteristics of those BIM uses. Shop drawings include detailed information about the component and the installation process since they are used in the production phase. FM task required detailed information related to the operation and maintenance phase including assembly code and the warranty period. Although the information that is required in the FM phase can be much more detailed based on the needs in the FM phase (East, 2007), this was not further investigated since it was out of the scope of this study.

The most frequently selected properties for both duct and pipe are the minimum modeling requirements for the defined BIM uses. Some properties are system derived and are automatically defined by the user when creating the component in the model (e.g. size, start and end offsets); however, most of them need to be specifically inputted by the user. It should also be noted that the level of effort to collect the required information to define each property might vary significantly. For example, more effort is required to collect "Assembly description" compared to "mark" property.

Although the information demand does not vary enormously for different BIM uses, it can vary significantly at different project types, for other BIM uses, construction components, or based on the requirements of the involved stakeholders. This change can influence the modeling time and effort considerably, as highlighted in the literature (Leite et al., 2011; Biljecki et al., 2016; Charef et al., 2018 \& Tan et al., 2019).

\section{VALIDATION}

To perform literal replication, six practitioners that had experience in transportation/infrastructure projects were divided into two groups (i.e., Group 1 and Group 2 in Table 1). The list of information items that were selected by the first group was compared with the list that was selected by the second group to check if the results were replicated. The aim was to determine how representative were the identified information requirements for practitioners having experience in the same project types. A property that was collectively selected by two groups (i.e., selected by at least one respondent in each of both groups) considered as a selected property among both groups. This comparison was performed for all the five BIM uses defined in the scope of this study. When the selected properties between the two groups were compared, the results showed that the list of information items that were identified by the first group was almost replicated by the second group or vice versa (Table 5). The ratio of literal replication ranged between $90 \%$ and $100 \%$ for duct and between $86 \%$ and $100 \%$ for pipe for all BIM uses. For example, the number of properties that were identified as necessary by the respondents in Group 1 for 4D simulation was 45 properties, and three out of these 45 properties were not selected by Group 2; therefore, the replication ratio is $95 \%$. This is the ratio of the number of replicated properties in two groups divided by the total number of properties. On the other hand, the respondents in Group 2 determined that 42 properties are required for $4 \mathrm{D}$ simulation and all the selected properties were also chosen by Group 1; therefore, the replication ratio is $100 \%$. The literal replication rates range from $91 \%$ to $100 \%$ for ducts and from $86 \%$ to $100 \%$ for pipes; hence, it can be confidently stated that the results are successfully replicated.

Table (5): Rates of literal replication among groups G1 and G2.

\begin{tabular}{|l|c|c|c|c|c|}
\hline \multicolumn{1}{|c|}{ Duct } & Clsh & 4D & QTO & ShD & FM \\
\hline \# of properties selected by G1 but not by G2 & 2 & 3 & 6 & 5 & 0 \\
\hline Replication ratio (1 - (items not selected by G2/total no. of items)*100) & $97 \%$ & $95 \%$ & $90 \%$ & $91 \%$ & $100.0 \%$ \\
\hline \# of properties selected by G2 but not by G1 & 4 & 0 & 2 & 0 & 5 \\
\hline Replication ratio (1 - (items not selected by G1/total no. of items)*100) & $93 \%$ & $100.0 \%$ & $97 \%$ & $100.0 \%$ & $91 \%$ \\
\hline \multicolumn{1}{|c|}{ Pipe } & Clsh & 4D & QTO & ShD & FM \\
\hline \# of properties selected by G1 but not by G2 & 4 & 4 & 6 & 6 & 0 \\
\hline Replication ratio 1 - (items missed by G2/total no. of items)*100 & $93 \%$ & $93 \%$ & $89 \%$ & $89 \%$ & $100.0 \%$ \\
\hline \# of properties selected by G2 but not by G1 & 8 & 0 & 4 & 2 & 6 \\
\hline Replication ratio 1 - (items missed by G1/total no. of items)*100 & $86 \%$ & $100.0 \%$ & $93 \%$ & $96 \%$ & $89 \%$ \\
\hline
\end{tabular}


The second stage was to test the external validity by comparing the combined results of the first two groups G1 and G2, that have experience in transportation/infrastructure projects, with the results of the third group G3, specialized in commercial/residential projects. The aim was to determine how representative were the identified information requirements for practitioners having experience in different project types. Similar to what was performed in the literal replication, if at least one respondent in group G1 or G2 choose a property and at the same time at least one respondent in G3 selected the same property, then this property is counted as an externally replicated property among the three groups $(\mathrm{G} 1, \mathrm{G} 2$, and G3).

When the results of the first two groups (i.e. G1 \& G2) were compared with the group (G3), the external validity rates were lower than literal replication rates (i.e., between $79 \%-91 \%$ for duct and between $75 \%-91 \%$ for pipe) as shown in Table 6. This was expected since the properties are being compared between two types of projects. The findings demonstrate that a higher number of properties were found to be ignored by G3's collective selections, meaning that respondents working at commercial/residential projects appear to be slightly less demanding for data while creating their BIM models. Specifically, 5 to 14 properties were ignored by G3 out of 58 and 56 total number of properties for both duct and pipe components respectively.

On the other hand, when the required properties of the respondents working in commercial/residential projects (i.e., G3) compared with that of the respondents experienced in transportation/infrastructure projects (i.e., G1 \& $\mathrm{G} 2$ ), it was revealed that information needed by the G3 was contained within the information required by the G1 and G2. i.e., for both duct and pipe components, the external validity ratio varied between $98 \%$ and $100 \%$, when G3 results were compared to the combined selections of groups G1 and G2 as shown in Table 6. This highlighted that more properties are required for transportation/infrastructure projects compared to commercial/residential projects for duct and pipe components and this can be associated with more complicated MEP systems in those types of projects.

The overall results of the external validation demonstrated that there are some differences in the required properties of duct and pipe components for different types of projects (i.e., transportation/infrastructure projects and commercial/residential projects); however, the majority of the required properties were found to be similar. Hence, it can be confidently stated that the properties that were identified are externally valid.

Table (6): External validity rates for duct and pipe items.

\begin{tabular}{|c|c|c|c|c|c|}
\hline Duct & Clsh & 4D & QTO & ShD & FM \\
\hline Missed by G3 (out of 58 properties) & 12 & 5 & 10 & 10 & 14 \\
\hline \multicolumn{6}{|c|}{ The high numbers mean the group (G3) is less demanding for information. } \\
\hline $\begin{array}{l}\text { External validity ratio } \\
1 \text { - (items missed by G3/total no. of items) } * 100\end{array}$ & $79 \%$ & $91 \%$ & $83 \%$ & $83 \%$ & $76 \%$ \\
\hline Missed by G1\&G2 & 0 & 0 & 1 & 0 & 0 \\
\hline $\begin{array}{l}\text { External validity ratio } \\
1 \text { - (items missed by G1\&G2/total no. of items) } * 100\end{array}$ & $100.0 \%$ & $100.0 \%$ & $98 \%$ & $100.0 \%$ & $100.0 \%$ \\
\hline Pipe & Clsh & 4D & QTO & ShD & FM \\
\hline Missed by G3 (out of 56 properties) & 14 & 5 & 12 & 10 & 13 \\
\hline \multicolumn{6}{|c|}{ The high numbers mean the group (G3) is less demanding for information. } \\
\hline $\begin{array}{l}\text { External validity ratio. } \\
1 \text { - (items missed by G3/total no. of items) } * 100\end{array}$ & $75 \%$ & $91 \%$ & $79 \%$ & $82 \%$ & $77 \%$ \\
\hline Missed by G1\&G2 & 0 & 0 & 1 & 0 & 0 \\
\hline $\begin{array}{l}\text { External validity ratio } \\
1 \text { - (items missed by } \mathrm{G} 1 \& \mathrm{G} 2 / \text { total no. of items) } * 100\end{array}$ & $100.0 \%$ & $100.0 \%$ & $98 \%$ & $100.0 \%$ & $100.0 \%$ \\
\hline
\end{tabular}

\section{CONCLUSIONS:}

An important step of BIM implementation is the definition of Level of Development (LOD), which describes the information items (i.e., geometric and non-geometric) to be included in a BIM model. This study identified the information items to be included in a BIM model based on the required BIM uses. The main sets of properties that were most frequently selected for two types of building components (i.e., duct and pipe) can be categorized under six groups: 1) constraints, 2) mechanical properties, 3) dimensions, 4) insulation, 5) lining (in Duct Case) and 6) accessories. These groups of properties represent the minimum modeling requirements. The categories of Identity Data, Mechanical Flow and Additional information were identified as the second-order groups of properties that can be added as required in other project types, or for other BIM uses.

BIM uses related to Shop Drawing (ShD) creation and Facility Management (FM) was found to be the most information demanding BIM uses due to their characteristics. Shop drawings include detailed information about 
the component and the installation process since they are used in the production phase. FM task required detailed information related to the operation and maintenance phase. Although the number of additionally required properties for these BIM uses is not that high for the components considered in this study, when the modeling effort for the entire discipline or project is considered, it might result in a significant increase. The findings also highlighted that more properties are required for transportation/infrastructure projects compared to commercial/residential projects for duct and pipe components and this can be an indication that more information is required for these types of projects due to more complicated MEP systems in transportation/infrastructure projects.

The list of identified information items required for representing ducts and pipes can be used by the practitioners when defining the properties required to prepare a BIM model for the selected BIM uses. Besides, the manufacturers and providers can use the findings of this study to define the minimum modeling essential information to be provided with their product's BIM files. This will also shift the modeling effort from the modeling team of the client, the designer, or the contractor, to the manufacturer's modeling team, which is the producer or developer of the component's model. Finally, the findings of the study can be used to improve the efficiency of the BIM adoption process and improve the decision-making capabilities of stakeholders in the AEC industry. It should be highlighted that the results are indicative due to the number of respondents and future research can consider reaching a larger number of practitioners in the field. Further research can be conducted on defining the information requirements for other types of components and other BIM uses. Also, automatically determining and entering the required information to the model is another research area. Artificial intelligence can be facilitated to capture the needed properties based on the types of spaces or relationships in the model under consideration.

\section{REFERENCES}

Abualdenien, J. and Borrmann, A., 2019. A meta-model approach for formal specification and consistent management of multi-LOD building models. Advanced Engineering Informatics, 40, pp.135-153.

Ahmad, A.M., Demian, P. and Price, A.D., 2012. Building information modelling implementation plans a comparative analysis.

AIA Document E202TM, 2008. Building Information Modeling Protocol Exhibit. The American Institute of Architects.

AIA, 2013. Guide, Instructions and Commentary to the 2013 AIA Digital Practice Documents (AIA Document E203-2013).

Alreshidi, E., Mourshed, M. and Rezgui, Y., 2017. Factors for effective BIM governance. Journal of Building Engineering, 10, pp.89-101.

Alreshidi, E., Mourshed, M. and Rezgui, Y., 2018. Requirements for cloud-based BIM governance solutions to facilitate team collaboration in construction projects. Requirements engineering, 23(1), pp.1-31.

Alshorafa, R. and Ergen, E., 2019. Determining the level of development for BIM implementation in large-scale projects. Engineering, Construction and Architectural Management.

Arayici, Y., Coates, P., Koskela, L., Kagioglou, M., Usher, C. and O'reilly, K., 2011. Technology adoption in the BIM implementation for lean architectural practice. Automation in construction, 20(2), pp.189-195.

Biljecki, F., Ledoux, H. and Stoter, J., 2016. An improved LOD specification for 3D building models. Computers, Environment and Urban Systems, 59, pp.25-37.

BIMForum, 2017. Level of Development Specification Guide.

BIMForum, 2019. Level of Development Specification Guide.

Boddy, C.R., 2016. Sample size for qualitative research. Qualitative Market Research: An International Journal.

BSI, 2013. PAS 1192-2:2013 Specification for information management for the capital / delivery phase of construction projects using building information modelling.

Charef, R., Alaka, H. and Emmitt, S., 2018. Beyond the third dimension of BIM: A systematic review of literature and assessment of professional views. Journal of Building Engineering, 19, pp.242-257.

Chong, H.Y., Lopez, R., Wang, J., Wang, X. and Zhao, Z., 2016. Comparative analysis on the adoption and use of BIM in road infrastructure projects. Journal of Management in Engineering, 32 (6), p.05016021. 
Computer Integrated Construction Research Group, 2011. Building Information Modeling Project Execution Planning Guide version-2.1. University Park: Pennsylvania State University.

Construction, M.H., 2014. The business value of BIM for construction in major global markets: How contractors around the world are driving innovation with building information modeling. Smart MarketReport.

Dakhil, A.J., Underwood, J. and Alshawi, M., 2019. Critical success competencies for the BIM implementation process: UK construction clients. Journal of Information Technology in Construction (ITcon), 24, pp.8094.

Department of Veterans Affairs. "The VA BIM Guide." US: US Department of Veterans, Version 1, (2010).

Eadie, R., Browne, M., Odeyinka, H., McKeown, C. and McNiff, S., 2013. BIM implementation throughout the UK construction project lifecycle: An analysis. Automation in construction, 36, pp.145-151.

East, E.W., 2007. Construction operations building information exchange (Cobie): Requirements definition and pilot implementation standard (No. ERDC/CERL TR-07-30). Engineer Research and Development Center Champaign IL Construction Engineering Research Lab.

Eastman, C.M., Eastman, C., Teicholz, P., Sacks, R. and Liston, K., 2011. BIM handbook: A guide to building information modeling for owners, managers, designers, engineers and contractors. John Wiley \& Sons.

EFCA, 2019. BIM and ISO 19650 from a project management perspective. (available online at: http://bimbooklet.efcanet.org/\#p=48 - last visited: 29/4/2020)

Flick, U., von Kardoff, E. and Steinke, I. eds., 2004. A companion to qualitative research. Sage.

Ghannadpour, S.F., RezaHoseini, A., Noori, S. and Yazdani, M., 2019. Analyzing the Influence of Building Information Modeling (BIM) on Construction Project Management Areas of Knowledge: Using a Hybrid FANP-FVIKOR Approach. International Journal of Industrial Engineering \& Production Research, 30(1), pp.57-92.

Gu, N. and London, K., 2010. Understanding and facilitating BIM adoption in the AEC industry. Automation in construction, 19(8), pp.988-999.

Herr, C.M. and Fischer, T., 2019. BIM adoption across the Chinese AEC industries: An extended BIM adoption model. Journal of Computational Design and Engineering, 6(2), pp.173-178.

Hong, Y., Hammad, A.W. and Akbarnezhad, A., 2019. Forecasting the net costs to organisations of Building Information Modelling (BIM) implementation at different levels of development (LOD). Journal of Information Technology in Construction (ITcon), 24(33), pp.588-603.

Ibrahim, N.H., 2013. Reviewing the evidence: use of digital collaboration technologies in major building and infrastructure projects. Journal of information technology in construction (ITcon), 18(3), pp.40-63.

ISO 2018 BS EN ISO 19650-1:2018. Organization and digitization of information about buildings and civil engineering works, including building information modelling (BIM) - Information management using building information modelling. Part 1: Concepts and principles.

Johnson, R.B., 1997. Examining the validity structure of qualitative research. Education, 118(2), p.282.

Jung, Y. and Joo, M., 2011. Building information modelling (BIM) framework for practical implementation. Automation in construction, 20(2), pp.126-133.

Kothari, C.R., 2004. Research methodology: Methods and techniques. New Age International.

Lai, H. and Deng, X., 2018. Interoperability analysis of IFC-based data exchange between heterogeneous BIM software. Journal of Civil Engineering and Management, 24(7), pp.537-555.

Latiffi, A.A., Brahim, J., Mohd, S. and Fathi, M.S., 2015. Building Information Modeling (BIM): Exploring Level of Development (LOD) in Construction Projects. Applied Mechanics and Materials, 773, p.933.

Leite, F., Akcamete, A., Akinci, B., Atasoy, G. and Kiziltas, S., 2011. Analysis of modeling effort and impact of different levels of detail in building information models. Automation in Construction, 20(5), pp.601-609.

Lin, Y.C., Chen, Y.P., Huang, W.T. and Hong, C.C., 2016. Development of BIM execution plan for bim model management during the pre-operation phase: a case study. Buildings, 6(1), p.8.

Liu, Y., Van Nederveen, S. and Hertogh, M., 2017. Understanding effects of BIM on collaborative design and construction: An empirical study in China. International Journal of Project Management, 35(4), pp.686698. 
Lu, Y., Wu, Z., Chang, R. and Li, Y., 2017. Building Information Modeling (BIM) for green buildings: A critical review and future directions. Automation in Construction, 83, pp.134-148.

Mzyece, D., Ndekugri, I.E. and Ankrah, N.A., 2019. Building information modelling (BIM) and the CDM regulations interoperability framework. Engineering, Construction and Architectural Management.

National BIM Specifications of Australia. "NATSPEC BIM Object-Element Matrix v1.0. (2011).

National Institute of Building Sciences buildingSMART alliance, 2015. National BIM Standard - United States, Version 3.

Nawari, N.O., 2012. BIM standard in off-site construction. Journal of Architectural Engineering, 18(2), pp.107113.

Olawumi, T.O. and Chan, D.W., 2019. Building information modelling and project information management framework for construction projects. Journal of Civil Engineering and Management, 25(1), pp.53-75.

Pezeshki, Z. and Ivari, S.A.S., 2018. Applications of BIM: a brief review and future outline. Archives of Computational Methods in Engineering, 25(2), pp.273-312.

Ritchie, J., Lewis, J., Nicholls, C.M. and Ormston, R. eds., 2013. Qualitative research practice: A guide for social science students and researchers. sage.

Shou, W., Wang, J., Wang, X. and Chong, H.Y., 2015. A comparative review of building information modelling implementation in building and infrastructure industries. Archives of computational methods in engineering, 22(2), pp.291-308.

Singapore, B.C.A., 2013. Singapore BIM Guide-Version 2. Singapore: Building and Construction Authority Singapore.

Singh, V., Gu, N. and Wang, X., 2011. A theoretical framework of a BIM-based multi-disciplinary collaboration platform. Automation in construction, 20(2), pp.134-144.

Succar, B., 2009. Building information modelling framework: A research and delivery foundation for industry stakeholders. Automation in construction, 18(3), pp.357-375.

Tan, T., Chen, K., Xue, F. and Lu, W., 2019. Barriers to Building Information Modeling (BIM) implementation in China's prefabricated construction: An interpretive structural modeling (ISM) approach. Journal of Cleaner Production, 219, pp.949-959.

Van Berlo, L.A.H.M. and Bomhof, F., 2014. Creating the Dutch national BIM levels of development. In Computing in Civil and Building Engineering (2014), pp. 129-136.

Vanlande, R., Nicolle, C. and Cruz, C., 2008. IFC and building lifecycle management. Automation in construction, 18(1), pp.70-78.

Wang, H. and Meng, X., 2018. BIM-Based Knowledge Management in Construction Projects. International Journal of Information Technology Project Management (IJITPM), 9(2), pp.20-37.

Wang, L. and Leite, F., 2015. Process knowledge capture in BIM-based mechanical, electrical, and plumbing design coordination meetings. Journal of Computing in Civil Engineering, 30(2), p.04015017.

Yalcinkaya, M. and Singh, V., 2019. Exploring the use of Gestalt's principles in improving the visualization, user experience and comprehension of COBie data extension. Engineering, Construction and Architectural Management.

Yin, Robert K., 2011. Qualitative research from start to finish, The Guilford Press, New York, p. 81. 